\title{
CHARACTERISTICES, CLASSIFICATION AND EVALUATION OF SOILS IN THE AREA SOUTHEAST EL-SADAT CITY, MENOUFIA GOVERNORATE, EGYPT
}

\author{
M.S. Amira (1), A.A. Shalaby (2), W.M. Omran ${ }^{(1)}$, and H.M. Elmedalaa ${ }^{(1)}$ \\ (1) Soil Sci. Dept., Fac. Agric. Menoufia univ . \\ (2) National Authority for Remote Sensing and Space Sciences (NARSS)
}

Received: Dec. 8, 2020

Accepted: Dec. 25, 2020

\begin{abstract}
The current work was performed in 2019 aiming to study the geomorphological and pedological characteristics as well as classification and capability evaluation for soils in the area southeastern El-Sadat City, Menoufia governorate, Egypt. The study area is located at the western side of the Nile-Delta in the east of CairoAlexandria desert highway adjacent to the Rashid branch of the River Nile, Menoufia governorate, Egypt. The integration of Remote Sensing (RS) and Geographic Information System (GIS) techniques was used to achieve this work.
\end{abstract}

The geomorphic map produced by processing and identifying the Landsat image using RS and GIS technology indicated that, the studied area has three main geomorphic units with different landforms. These three main units are 1) Alluvial Plain includes three landforms namely: High Terraces (17.33\% of the studied area), Moderate Terraces (5.87\%) and Low Terraces (11.48\%); 2) Pediplain with two landforms namely: High Terraces (16.22 \%) and Low Terraces $(12.47 \%) ; 3$ ) as well as Aeolian Plain with three landforms namely: High Terraces (7.08\%), Moderate Terraces (4.97\%) and Low Terraces $(24.58 \%)$.

Nineteen soil profiles were chosen to represent the different geomorphic and landform units. The land and site features are observed and registered. The soil profiles were dug, morphologically described, and then samples were collected representing the subsequent layers in each profile for integrated physical and chemical analyses. Also, irrigation water samples were collected and analyzed especially for land capability evaluation.

The studied area has almost flat to slightly undulated topography. Soils were deep to very deep where they were well drained. Most of the soils have gravely sand to gravely loamy sand texture except the soils of low Alluvial plain terraces that have clay loam texture.

The analytical data revealed that, the studied soils are slightly to moderately alkaline, mostly non-saline and haven't sodicity effect. Few parts showed a sodic horizon feature. The soils are slightly to moderately calcareous having Low gypsum and Organic matter contents.

Most of the soils haven't any diagnostic horizons, therefore they were classified under Entisols. The few soils having sodic horizon were classified under Aridisols.

The land capability evaluation indicated that, most of the studied soils (92.18\%) are affiliated to the Fair, C3 class. Rest of the soils are classified either as a Good capability class, C2, (4.52\%), or as a Poor capability class, C4 (3.30\%).

Key words: RS, GIS, geomorphic units, landforms, pedological features, soil classification, land capability evaluation. 


\section{INTRODUCTION}

The balance between the land and human resources is the most critical problem in Egypt. Accordingly, the major challenge of Egyptian government today is facing the need for better development and management of natural resources, to meet requirements of the fast-growing population. Therefore, agriculture expansion in the Western Desert is one of the most vital objectives in Egyptian policy to satisfy the food security needs of the ever- increasing population (Ismail et al., 2010). One of the susceptible lands occurs mainly in the fringes of the Nile valley and Delta. Fringes of the Nile Delta are considered to be the most important location of the ambitious projects. These fringes have the most of best potentially suitable agricultural land resources for future expansion and development in Egypt.

El-Sadat City area is located at the northeastern side of the Western Desert of Egypt to the west of Nile Delta and the east of Cairo-Alexandria desert road adjacent to the Rosetta branch of the River Nile. It is generally formed from some low-lying hills that received special attention due to its reasonably good groundwater resources.

Geomorphologically, this area in the western side of Nile Delta is divided into four units; young alluvial plains, old alluvial plains, conglomerates and sand dunes (Dawoud et al., 2005).

El-Maaz (1997) stated that, all the studied soils in western side of the Nile Delta have Torric soil moisture regime with Thermic soil temperature regime. She classified these soils according Soil Survey staff (1994) as: a) Typic Torripsamments and Typic Torriorthents.

The western Nile Delta contains four aquifer systems: Delta aquifer (Quaternary), Wadi El Natrun aquifer (Pliocene), El Moghra aquifer (Miocene), and the Oligocene aquifer (RIGW and IWACO, 1991).

Remote sensing (RS) is now recognized as an important tool in monitoring and managing natural resources (Lillesand and Kiefer, 2007). They added that RS technique is one of the important methods that used for soil survey, mapping and environmental investigation.

ESRI (2003) stated that, geographic information system (GIS) is a system for the management, analysis, and displaying geographic information, which is represented by a series of geographic datasets that model geography using simple, generic data structures.

Integration of RS and GIS play a major role in both soil survey and soil mapping applications. The development of methods to map soil properties using optical RS data in combination with field measurements has been the objective of several studies during the last decade (Dehaan and Taylor, 2003).

The aim of the present work is to furnish a comprehensive study on geomorphological and pedological features of the soils in the area southeast of El-Sadat City using the integration of Remote Sensing (RS) and Geographic Information System (GIS) techniques. Also, soil classification and land capability evaluation are achieved.

\section{MATERIALS AND METHODS}

\section{Study area}

The study area is located at the southeastern part of El-Sadat City, in the east of Cairo-Alexandria desert highway. It lies between longitudes $30^{\circ} 34^{\prime} 00^{\prime \prime}-$ $30^{\circ} 52^{\prime} 00^{\prime \prime} E$ and latitudes $30^{\circ} 16^{\prime} 00^{\prime \prime}-$ $30^{\circ} 28^{\prime} 00^{\prime \prime} \mathrm{N}$ covering an area of $353.29 \mathrm{~km}^{2}$ (84117.92 Feddan), Fig (1). The studied area is characterized by a hot dry summer and warm winter with few rainfalls. 


\section{Geomorphology of the study area}

The digital elevation model (DEM) of the study area was extracted from the Shuttle Radar Topography Mission (SRTM) and a topographic map with a scale of $1: 25,000$ covering the study area using Arc-GIS 10.4.1 software (ESRI, 2003). The Landsat 8 (path 178 I row 39) image acquired in 2019 and SRTM data were processed in ENVI 5.1 software (ITT, 2012) to identify the geomorphology and landforms of the studied area according to the approach developed by Dobos et al. (2002). The map legend was designed according to Zinck and Valenzuela (1990). ArcMap 10.4.1 software was used to display and produce geomorphic map of the study area with help of its DEM features and field observations (ESRI, 2014).

\section{Field work.}

Reconnaissance soil survey was conducted throughout the investigated area in order to acquire an appreciation of its broad soil patterns and characteristic landscape. The primary mapping units resulting from analysis of the DEM and interpretation information gained during unsupervised classification Landsat images were verified.

Longitudes, latitudes, elevations and soil profiles locations were defined in the field by using GPS system "Corporation MAGELLAN"- NAV DLX-10 TM.

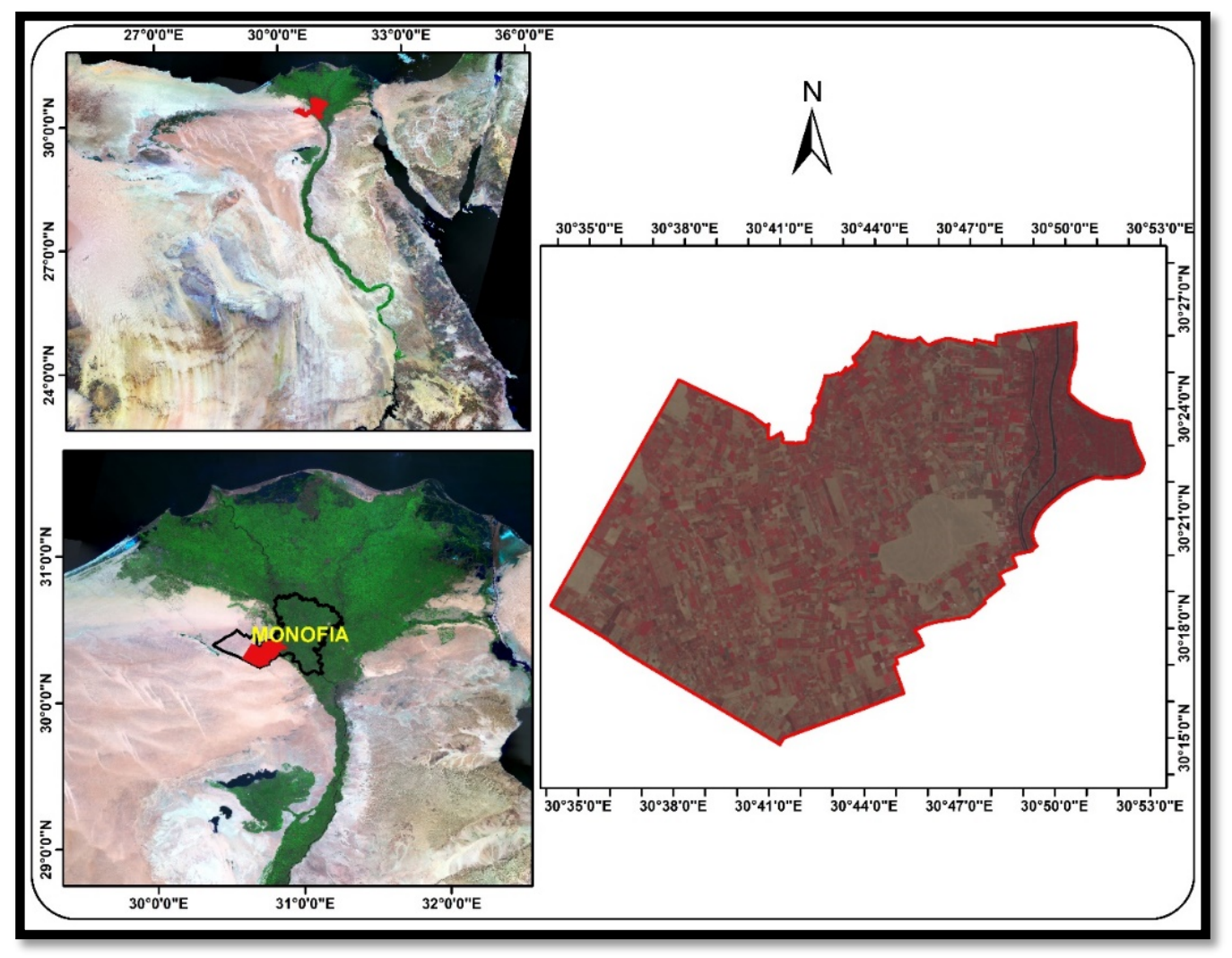

Fig (1): Location map of study area. 
Nineteen soil profiles were chosen to represent the landform units in the studied area (Fig, 2). Detailed morphological description of these soil profiles were recorded on the basis outlined by FAO (2006). Soil samples were collected based on the vertical variations of each soil profile for the laboratory analyses of soil physical and chemical properties. Also, water samples were collected from the available irrigation water sources for laboratory analyses of their chemical properties that used in the model of land capability evaluation.

\section{Laboratory analyses}

Particle size distribution, electrical conductivity (EC), pH, organic matter (OM), calcium carbonate $\left(\mathrm{CaCO}_{3}\right)$, gypsum contents, cation exchange capacity (CEC), hydraulic conductivity (HC), exchangeable $\mathrm{Na}^{+}$percentage (ESP) and available $N, P$ and $K$ in the soil surface samples were determined according to Burt and Soil Survey Staff (2014). The weighted profile mean (w.p.m.) of each soil property was calculated for the studied profiles. Also, EC, pH, soluble cations and anions as well as sodium adsorption ratio (SAR) and soluble boron were determined in the irrigation water samples according to Burt and Soil Survey Staff (2014).

\section{Soil classification}

The soils of the studied area were classified up to sub great group level based on Soil Survey Staff (2014).

\section{Land Evaluation}

Land capability classification was carried out using the Agriculture Land Evaluation System for arid and semi-arid regions (ALES-Arid) developed by Ismail et al. (1994 and 2005). ALES-Arid software is inserting of soil database and calculates possible indices combinations between the major land properties. These properties are, soil physical properties (clay content, available water, profile depth, landform, slope and level of surface), chemical properties $(\mathrm{pH}$, soil salinity, gypsum and carbonate contents), soil fertility (OM, available $N, P$, and $K$ ) and Irrigation water characteristics $(\mathrm{pH}, \mathrm{EC}$, SAR and soluble boron).

\section{RESULTUS AND DISCUSSION Geomorphology}

Based on the satellite image treating, processing and interpretation with the help of topographic maps and field survey, the integration of RS and GIS was used to identify the geomorphic and landform units of the study area. The main interpreted and identified geomorphic units in the investigated area are Alluvial Plain, Pediplain and Aeolian Plain with detailed gradated landforms as shown with their representative soil profiles in Fig (2) and Table (1).

\section{Soil morphology}

The morphological features of the studied soils presented in Table (2) revealed that, the elevation of the studied soils is between $10 \mathrm{~m}$ to $90 \mathrm{~m}$ above sea level. The soils have almost flat to slightly undulating topography. Most of studied soils are very deep and well drained. The main hue notation of studied soil color is around brown to red degrees (10YR to 7,5YR). The soils have mostly weak to moderate medium to fine subangular blocky and granular structure. The sandy soils have single grains. The studied soils are slightly to moderately calcareous having mostly slightly hard (dry) and firm (moist) consistency. The most studied soils are cultivated with field or horticultural (vegetable and/or fruit) crops. 


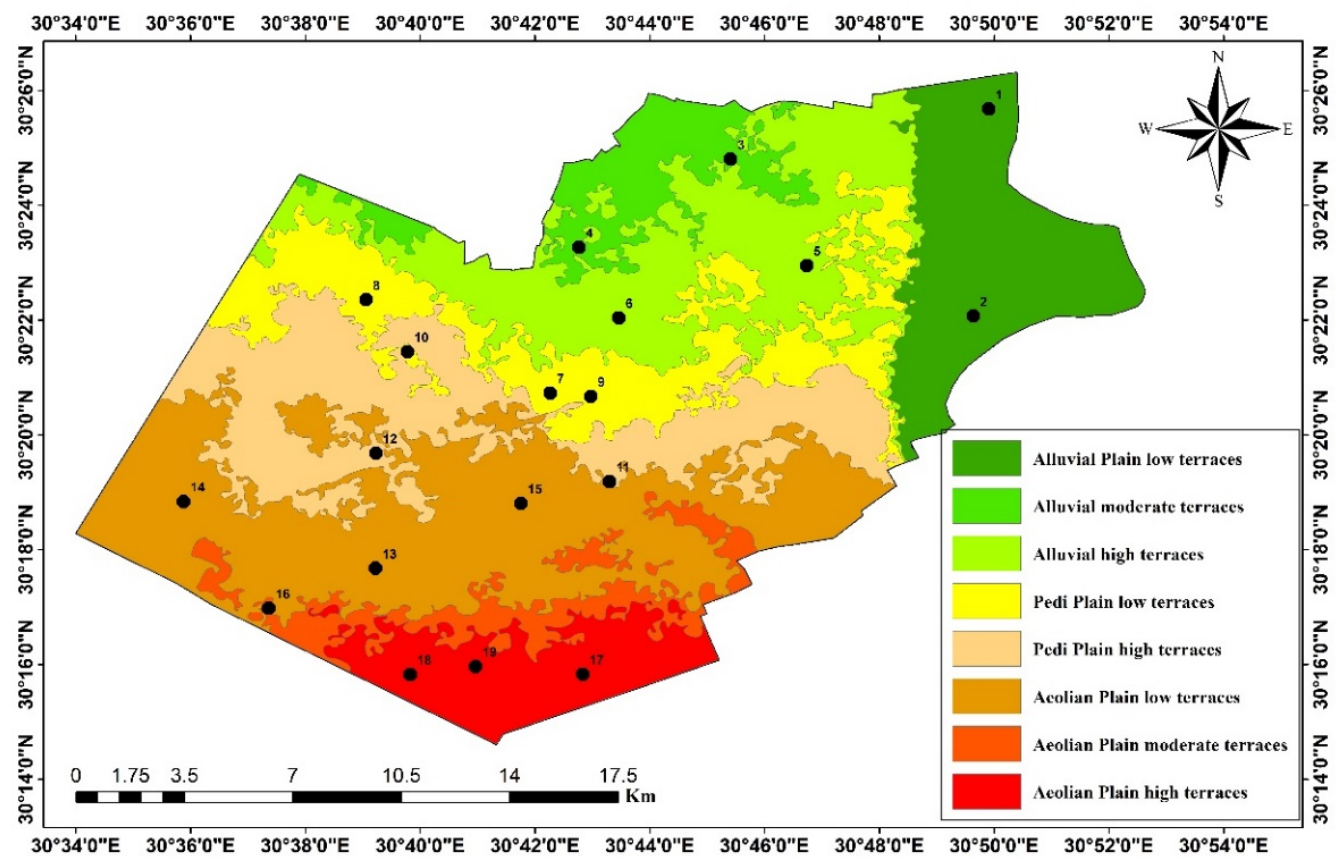

Fig (2): Detailed geomorphic map and locations of soil profiles in the study area.

Table (1): Geomorphic and landform units of the study area and their profiles and areas.

\begin{tabular}{|c|c|c|c|c|}
\hline \multirow{2}{*}{ Geomorphic units } & \multirow{2}{*}{ landform } & \multirow{2}{*}{ Profiles No. } & \multicolumn{2}{|c|}{ Area in } \\
\hline & & & Feddan & $\%$ \\
\hline \multirow{4}{*}{ Alluvial Plain } & Low Terraces & P1, P2 & 9654.55 & 11.48 \\
\hline & Moderate Terraces & P3, P4 & 4938.99 & 5.87 \\
\hline & High Terraces & P5, P6 & 14581.78 & 17.33 \\
\hline & \multicolumn{2}{|c|}{ Total } & 29175.32 & 34.68 \\
\hline \multirow{3}{*}{ Pediplain } & Low Terraces & P7, P8, P9 & 10486.53 & 12.47 \\
\hline & High Terraces & P10, P11, P12 & 13641.04 & 16.22 \\
\hline & \multicolumn{2}{|c|}{ Total } & 24127.57 & 28.69 \\
\hline \multirow{4}{*}{ Aeolian Plain } & Low Terraces & P13, P14, P15 & 20679.88 & 24.58 \\
\hline & Moderate Terraces & P16 & 4183.02 & 4.97 \\
\hline & High Terraces & P17, P18, P19 & 5952.13 & 7.08 \\
\hline & \multicolumn{2}{|c|}{ Total } & 30815.03 & 36.63 \\
\hline \multicolumn{3}{|c|}{ Total of Study area } & 84117.92 & 100 \\
\hline
\end{tabular}


M.S. Amira, et al.,

Table (2): Morphological features of the studied soil profiles.

\begin{tabular}{|c|c|c|c|c|c|c|c|c|c|c|}
\hline \multirow{2}{*}{ 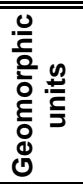 } & \multirow{2}{*}{ 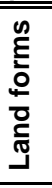 } & \multirow[b]{2}{*}{$\begin{array}{c}\text { Profile } \\
\text { No. }\end{array}$} & \multirow[b]{2}{*}{ 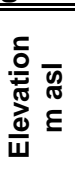 } & \multirow[b]{2}{*}{ Depth cm } & \multicolumn{2}{|c|}{ Color } & \multirow[b]{2}{*}{ Structure $^{1}$} & \multicolumn{2}{|c|}{ Consistence $^{2}$} & \multirow[b]{2}{*}{ Boundary $^{3}$} \\
\hline & & & & & Dry & Moist & & Dry & Moist & \\
\hline \multirow{6}{*}{ 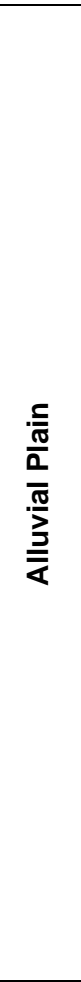 } & \multirow{2}{*}{ 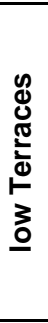 } & 1 & +10 & \begin{tabular}{|c|}
$0-30$ \\
$30-60$ \\
$60-90$ \\
$90-110$ \\
$110-130$ \\
\end{tabular} & $\begin{array}{l}\text { 10YR 5/2 } \\
\text { 10YR 5/2 } \\
\text { 10YR 5/2 } \\
\text { 10YR 5/2 } \\
\text { 10YR 5/3 }\end{array}$ & $\begin{array}{l}3 / 2 \\
4 / 2 \\
3 / 2 \\
4 / 2 \\
4 / 3\end{array}$ & $\begin{array}{l}2 \mathrm{c} \text { to } \mathrm{m} \mathrm{sbk} \\
2 \mathrm{c} \text { to } \mathrm{sbk} \\
2 \mathrm{c} \text { to } \mathrm{sbk} \\
2 \mathrm{c} \text { to } \mathrm{fbbk} \\
1 \mathrm{~m} \text { to } \mathrm{s} s \mathrm{sk}\end{array}$ & $\begin{array}{l}\text { ex hard } \\
\text { ex hard } \\
\text { ex hard } \\
\text { ex hard } \\
\text { ex hard }\end{array}$ & $\begin{array}{l}\text { v firm } \\
\text { ex firm } \\
v \text { firm } \\
\text { firm } \\
\text { friable }\end{array}$ & $\begin{array}{c}\text { diffuse } \\
\text { diffuse } \\
\text { gradual s } \\
\text { diffuse } \\
-\end{array}$ \\
\hline & & 2 & +17 & \begin{tabular}{|c|}
$0-30$ \\
$30-60$ \\
$60-90$ \\
$90-110$ \\
$110-130$ \\
\end{tabular} & $\begin{array}{l}\text { 10YR 4/2 } \\
\text { 10YR 5/2 } \\
\text { 10YR 5/2 } \\
\text { 10YR 5/2 } \\
\text { 10YR 5/3 }\end{array}$ & $\begin{array}{l}3 / 2 \\
4 / 2 \\
3 / 2 \\
4 / 2 \\
4 / 3\end{array}$ & $\begin{array}{c}2 \mathrm{c}, \mathrm{m} \text { to } \mathrm{f} \mathrm{sbk} \\
2 \mathrm{c} \text { to } \mathrm{fbk} \\
2 \mathrm{c} \text { to } \mathrm{fbbk} \\
2 \mathrm{c} \text { to } \mathrm{f} s \mathrm{kk} \\
1 \mathrm{~m} \text { to } \mathrm{sbk}\end{array}$ & $\begin{array}{l}\text { V hard } \\
\text { ex hard } \\
\text { ex hard } \\
\text { ex hard } \\
\text { ex hard }\end{array}$ & \begin{tabular}{|c|} 
firm \\
ex firm \\
v firm \\
firm \\
friable
\end{tabular} & $\begin{array}{c}\text { gradual s } \\
\text { diffuse } \\
\text { gradual s } \\
\text { diffuse } \\
-\end{array}$ \\
\hline & \multirow{2}{*}{ 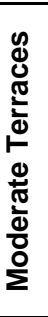 } & 3 & +33 & $\begin{array}{c}0-30 \\
30-60 \\
60-90 \\
90-140 \\
\end{array}$ & $\begin{array}{l}\text { 10YR 6/4 } \\
\text { 10YR 6/5 } \\
\text { 10YR 7/4 } \\
\text { 10YR 7/4 } \\
\end{array}$ & $\begin{array}{l}5 / 4 \\
5 / 5 \\
3 / 2 \\
6 / 4 \\
\end{array}$ & $\begin{array}{c}1 \mathrm{~m} \text { to f sbk } \\
1 \mathrm{gr}+\mathrm{sg} \\
\mathrm{sg} \\
\mathrm{sg}\end{array}$ & $\begin{array}{l}\text { s hard } \\
\text { loose } \\
\text { loose } \\
\text { loose } \\
\end{array}$ & $\begin{array}{l}\text { Friable } \\
\text { loose } \\
\text { loose } \\
\text { loose } \\
\end{array}$ & $\begin{array}{c}\text { gradual s } \\
\text { gradual s } \\
\text { diffuse } \\
- \\
\end{array}$ \\
\hline & & 4 & +33 & $\begin{array}{c}0-30 \\
30-60 \\
60-90 \\
90-120 \\
120-150\end{array}$ & $\begin{array}{l}\text { 10YR 5/6 } \\
7.5 Y R 5 / 6 \\
\text { 10YR 6/6 } \\
\text { 10YR 6/6 } \\
\text { 10YR 6/6 }\end{array}$ & $\begin{array}{l}4 / 6 \\
4 / 6 \\
5 / 6 \\
5 / 6 \\
5 / 6\end{array}$ & $\begin{array}{c}2 \mathrm{~m} \text { to } f \mathrm{sbk} \\
2 \mathrm{~m} \text { to } \mathrm{s} s \mathrm{k} \\
1 \mathrm{~m} \text { to } \mathrm{s} \mathrm{sbk} \\
1 \mathrm{~m} \text { to } \mathrm{f} \mathrm{sbk} \\
1 \mathrm{fsbk}\end{array}$ & $\begin{array}{l}\text { s hard } \\
\text { s hard } \\
\text { soft } \\
\text { soft } \\
\text { soft }\end{array}$ & $\begin{array}{l}\text { friable } \\
\text { friable } \\
\text { loose } \\
\text { loose } \\
\text { loose }\end{array}$ & $\begin{array}{c}\text { gradual s } \\
\text { clear } \\
\text { diffuse } \\
\text { diffuse } \\
-\end{array}$ \\
\hline & \multirow{2}{*}{ 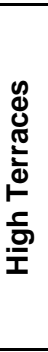 } & 5 & +41 & $\begin{array}{c}0-30 \\
30-60 \\
60-90 \\
90-120 \\
120-150 \\
\end{array}$ & $\begin{array}{l}\text { 10YR 5/6 } \\
7.5 Y R \text { 5/6 } \\
\text { 10YR 6/6 } \\
\text { 10YR 6/6 } \\
\text { 10YR 6/6 } \\
\end{array}$ & $\begin{array}{l}4 / 6 \\
4 / 6 \\
5 / 6 \\
5 / 6 \\
5 / 6 \\
\end{array}$ & $\begin{array}{c}2 \mathrm{~m} \text { to } f \mathrm{sbk} \\
2 \mathrm{~m} \text { to } \mathrm{f} \mathrm{sbk} \\
1 \mathrm{~m} \text { to } \mathrm{f} \mathrm{sbk} \\
1 \mathrm{~m} \text { to } \mathrm{f} \mathrm{sbk} \\
1 \mathrm{fsbk}\end{array}$ & $\begin{array}{l}\text { S hard } \\
\text { s hard } \\
\text { soft } \\
\text { soft } \\
\text { soft } \\
\end{array}$ & $\begin{array}{l}\text { loose } \\
\text { loose } \\
\text { loose } \\
\text { loose } \\
\text { loose } \\
\end{array}$ & $\begin{array}{l}\text { diffuse } \\
\text { diffuse } \\
\text { diffuse } \\
\text { diffuse } \\
\text { - } \\
\end{array}$ \\
\hline & & 6 & +39 & $\begin{array}{c}0-30 \\
30-60 \\
60-90 \\
90-120 \\
120-150\end{array}$ & $\begin{array}{l}\text { 10YR 6/6 } \\
\text { 10YR 6/6 } \\
\text { 10YR 6/5 } \\
\text { 10YR 6/5 } \\
\text { 10YR 6/5 }\end{array}$ & $\begin{array}{l}5 / 6 \\
5 / 6 \\
5 / 5 \\
5 / 5 \\
5 / 5\end{array}$ & $\begin{array}{c}2 \mathrm{~m} \text { to } f s b k \\
2 \mathrm{~m} \text { to } f s b k \\
2 \mathrm{~m} \text { to } f s b k \\
2 \mathrm{~m} \text { to } f s b k \\
1 \mathrm{f} \mathrm{sbk}\end{array}$ & $\begin{array}{l}\text { s hard } \\
\text { s hard } \\
\text { s hard } \\
\text { s hard } \\
\text { s hard }\end{array}$ & $\begin{array}{c}\text { friable } \\
\text { friable } \\
\text { friable } \\
\text { friable } \\
\text { friable }\end{array}$ & $\begin{array}{c}\text { diffuse } \\
\text { gradual s } \\
\text { diffuse } \\
\text { diffuse } \\
-\end{array}$ \\
\hline \multirow{6}{*}{$\frac{\frac{5}{\pi}}{\frac{\pi}{\frac{2}{2}}}$} & \multirow{3}{*}{ 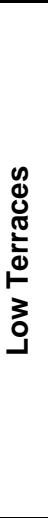 } & 7 & +50 & $\begin{array}{c}0-30 \\
30-60 \\
60-90 \\
90-120 \\
120150 \\
\end{array}$ & $\begin{array}{l}\text { 10YR 6/6 } \\
7.5 Y R 6 / 5 \\
7.5 Y R 6 / 5 \\
7.5 Y R 6 / 5 \\
7.5 Y R 6 / 4 \\
\end{array}$ & $\begin{array}{l}5 / 6 \\
5 / 5 \\
5 / 5 \\
5 / 5 \\
5 / 4 \\
\end{array}$ & $\begin{array}{l}2 \mathrm{c} \text { to } f \mathrm{sbk} \\
2 \mathrm{~m} \text { to } \mathrm{f} \mathrm{sbk} \\
2 \mathrm{~m} \text { to } f \mathrm{sbk} \\
2 \mathrm{~m} \text { to } \mathrm{sbk} \\
2 \mathrm{~m} \text { to } \mathrm{s} s \mathrm{k}\end{array}$ & $\begin{array}{l}\text { s hard } \\
\text { s hard } \\
\text { s hard } \\
\text { s hard } \\
\text { loose } \\
\end{array}$ & $\begin{array}{c}\text { Friable } \\
\text { Friable } \\
\text { friable } \\
\text { friable } \\
\text { loose } \\
\end{array}$ & $\begin{array}{l}\text { clear } \\
\text { diffuse } \\
\text { diffuse } \\
\text { diffuse } \\
- \\
\end{array}$ \\
\hline & & 8 & +49 & \begin{tabular}{|c|}
$0-25$ \\
$25-45$ \\
$45-77$ \\
$77-105$ \\
$105-150$ \\
\end{tabular} & $\begin{array}{l}\text { 7.5YR 7/6 } \\
\text { 10YR 5/2 } \\
\text { 10YR 6/6 } \\
\text { 10YR 6/6 } \\
7.5 Y R ~ 6 / 6 \\
\end{array}$ & $\begin{array}{l}6 / 6 \\
3 / 2 \\
5 / 6 \\
5 / 6 \\
5 / 6 \\
\end{array}$ & $\begin{array}{l}2 \mathrm{~m} \text { to } f \mathrm{sbk} \\
2 \mathrm{~m} \text { to } \mathrm{sbk} \\
2 \mathrm{~m} \text { to } \mathrm{sbk} \\
2 \mathrm{~m} \text { to } \mathrm{s} s \mathrm{k} \\
2 \mathrm{~m} \text { to } \mathrm{sbl}\end{array}$ & $\begin{array}{l}\text { s hard } \\
\text { s hard } \\
\text { s hard } \\
\text { s hard } \\
\text { s hard }\end{array}$ & $\begin{array}{c}\text { friable } \\
\text { friable } \\
\text { friable } \\
\text { friable } \\
\text { friable }\end{array}$ & $\begin{array}{l}\text { abrupt } \\
\text { abrupt } \\
\text { diffuse } \\
\text { diffuse } \\
-\end{array}$ \\
\hline & & 9 & +49 & \begin{tabular}{|c|}
$0-30$ \\
$30-60$ \\
$60-90$ \\
$90-120$ \\
$120-150$ \\
\end{tabular} & $\begin{array}{l}\text { 10YR 6/6 } \\
\text { 7.5YR 6/4 } \\
\text { 10YR 6/5 } \\
\text { 10YR 6/4 } \\
\text { 10YR 6/4 } \\
\end{array}$ & $\begin{array}{l}5 / 6 \\
5 / 4 \\
5 / 5 \\
5 / 4 \\
5 / 4\end{array}$ & $\begin{array}{c}2 \mathrm{~m} \text { to f sbk } \\
2 \mathrm{~m} \text { to f sbk } \\
1 \mathrm{f} \mathrm{sbk} \\
\mathrm{sg} \\
1 \mathrm{f} \mathrm{sbk}+\mathrm{sg}\end{array}$ & $\begin{array}{l}\text { s hard } \\
\text { s hard } \\
\text { s hard } \\
\text { loose } \\
\text { soft }\end{array}$ & $\begin{array}{c}\text { friable } \\
\text { friable } \\
\text { friable } \\
\text { loose } \\
\text { v friable }\end{array}$ & $\begin{array}{c}\text { gradual s } \\
\text { gradual s } \\
\text { diffuse } \\
\text { diffuse } \\
- \\
\end{array}$ \\
\hline & \multirow{3}{*}{ 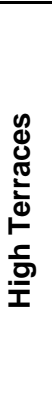 } & 10 & +52 & $\begin{array}{c}0-50 \\
50-70 \\
70-110 \\
110-150 \\
\end{array}$ & $\begin{array}{l}\text { 10YR 6/5 } \\
7.5 Y R \text { 7/5 } \\
\text { 10YR 6/5 } \\
7.5 Y R \text { 5/6 } \\
\end{array}$ & $\begin{array}{l}5 / 5 \\
6 / 5 \\
5 / 5 \\
4 / 6 \\
\end{array}$ & $\begin{array}{c}\mathrm{Sg} \\
1 \mathrm{~m} \text { to } \mathrm{f} \mathrm{sbk} \\
2 \mathrm{c}, \mathrm{m} \text { to } \mathrm{f} \text { sbk } \\
\mathrm{sg}\end{array}$ & $\begin{array}{l}\text { loose } \\
\text { s hard } \\
\text { ex hard } \\
\text { loose }\end{array}$ & $\begin{array}{c}\text { loose } \\
\text { friable } \\
\text { friable } \\
\text { loose } \\
\end{array}$ & $\begin{array}{c}\text { gradual s } \\
\text { clear } \\
\text { abrupt } \\
-\end{array}$ \\
\hline & & 11 & +56 & $\begin{array}{c}0-30 \\
30-50 \\
50-90 \\
90-150\end{array}$ & $\begin{array}{l}7.5 Y R 6 / 5 \\
\text { 10YR 5/3 } \\
\text { 10YR 6/6 } \\
7.5 Y R \text { 6/5 }\end{array}$ & $\begin{array}{l}5 / 5 \\
4 / 3 \\
5 / 6 \\
5 / 5\end{array}$ & $\begin{array}{l}1 \mathrm{~m} \text { to } f \mathrm{sbk} \\
2 \mathrm{~m} \text { to } \mathrm{sbk} \\
2 \mathrm{c} \text { to } \mathrm{m} \mathrm{bk} \\
2 \mathrm{~m} \text { to } \mathrm{fbbk}\end{array}$ & $\begin{array}{l}\text { s hard } \\
\text { s hard } \\
\text { hard } \\
\text { s hard }\end{array}$ & $\begin{array}{c}\text { Friable } \\
\text { Friable } \\
\text { friable } \\
\text { friable }\end{array}$ & $\begin{array}{c}\text { abrupt } \\
\text { abrupt } \\
\text { gradual s } \\
-\end{array}$ \\
\hline & & 12 & +59 & $\begin{array}{c}0-20 \\
20-70 \\
70-100 \\
100-140\end{array}$ & $\begin{array}{l}7.5 Y R \text { 6/6 } \\
7.5 Y R \text { 7/6 } \\
\text { 10YR 6/3 } \\
7.5 Y R 6 / 6\end{array}$ & $\begin{array}{l}5 / 6 \\
5 / 6 \\
4 / 3 \\
5 / 6\end{array}$ & $\begin{array}{c}1 \mathrm{~m} \text { to } \mathrm{f} \mathrm{gr} \\
2 \mathrm{~m} \text { to } \mathrm{f} \mathrm{sbk} \\
2 \mathrm{~m} \text { to } \mathrm{f} \mathrm{sbk} \\
\mathrm{sg}\end{array}$ & $\begin{array}{l}\text { s hard } \\
v \text { hard } \\
\text { ex hard } \\
\text { loose }\end{array}$ & $\begin{array}{c}\text { Friable } \\
\text { friable } \\
\text { friable } \\
\text { loose }\end{array}$ & $\begin{array}{c}\text { gradual s } \\
\text { abrupt } \\
\text { abrupt } \\
-\end{array}$ \\
\hline
\end{tabular}

Abbreviations: Texture*: L=loamy, $\mathrm{S}=$ sandy, $\mathrm{s} \mathbf{g}=$ =slightly gravely, $\mathrm{g}=$ gravely; Structure ${ }^{1}:$ 1=weak, 2 =moderate $v=$ very, $\mathrm{f}=$ fine, $\mathrm{m}=$ medium, $\mathrm{c}=$ coarse, $\mathrm{gr}=$ granular, $\mathrm{sbk}=$ subangular blocky, $\mathrm{bk}=$ blocky, $\mathrm{sg}=$ single grains; Consistence ${ }^{2}: \mathrm{v}=$ very, $\mathrm{x}=$ extremely; Boundary ${ }^{3}: \mathrm{s}=$ smooth 
Table (2): Content.

\begin{tabular}{|c|c|c|c|c|c|c|c|c|c|c|}
\hline \multirow{2}{*}{ 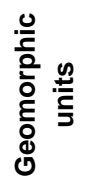 } & \multirow{2}{*}{ 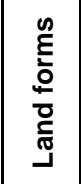 } & \multirow[b]{2}{*}{$\begin{array}{c}\text { Profile } \\
\text { No. }\end{array}$} & \multirow{2}{*}{ 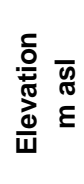 } & \multirow[b]{2}{*}{ Depth cm } & \multicolumn{2}{|c|}{ Color } & \multirow[b]{2}{*}{ Structure $^{1}$} & \multicolumn{2}{|c|}{ Consistence $^{2}$} & \multirow[b]{2}{*}{ Boundary } \\
\hline & & & & & Dry & Moist & & Dry & Moist & \\
\hline \multirow{7}{*}{ 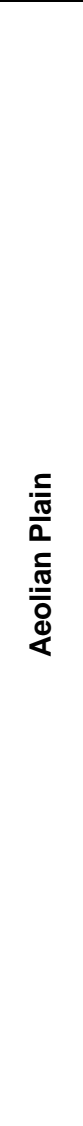 } & \multirow{3}{*}{ 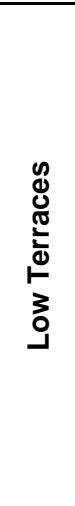 } & 13 & +59 & $\begin{array}{c}0-20 \\
20-40 \\
40-100 \\
100-110 \\
110-150 \\
\end{array}$ & $\begin{array}{l}\text { 10YR 6/4 } \\
\text { 10YR 6/6 } \\
\text { 7.5YR 6/6 } \\
7.5 Y R 6 / 6 \\
7.5 Y R ~ 6 / 6 \\
\end{array}$ & $\begin{array}{l}5 / 4 \\
5 / 6 \\
5 / 6 \\
5 / 6 \\
5 / 6\end{array}$ & $\begin{array}{l}1 \mathrm{f} \mathrm{gr} \\
1 \mathrm{~m} \text { to f sbk } \\
1 \mathrm{~m} \text { to f sbk } \\
1 \mathrm{~m} \text { to } \mathrm{f} \mathrm{sbk} \\
1 \mathrm{f} \mathrm{sbk} \text { to } \mathrm{gr}\end{array}$ & $\begin{array}{l}\text { s hard } \\
s \text { hard } \\
s \text { hard } \\
s \text { hard } \\
s \text { hard }\end{array}$ & $\begin{array}{l}\text { friable } \\
\text { friable } \\
\text { friable } \\
\text { friable } \\
\text { friable } \\
\end{array}$ & $\begin{array}{c}\text { gradual s } \\
\text { abrupt } \\
\text { abrupt } \\
\text { abrupt } \\
- \\
\end{array}$ \\
\hline & & 14 & +55 & $\begin{array}{c}0-30 \\
30-60 \\
60-90 \\
90-120 \\
120-150\end{array}$ & $\begin{array}{l}\text { 10YR 6/6 } \\
7.5 Y R 6 / 6 \\
7.5 Y R 6 / 6 \\
\text { 10YR 7/7 } \\
7.5 Y R 7 / 6\end{array}$ & $\begin{array}{l}5 / 6 \\
5 / 6 \\
5 / 6 \\
6 / 7 \\
6 / 6\end{array}$ & $\begin{array}{l}2 \mathrm{~m} \text { to } f \mathrm{sbk} \\
2 \mathrm{~m} \text { to } \mathrm{sbk} \\
2 \mathrm{~m} \text { to } f \mathrm{sbk} \\
2 \mathrm{~m} \text { to } \mathrm{sbk} \\
2 \mathrm{~m} \text { to } \mathrm{sbk}\end{array}$ & $\begin{array}{l}\text { s hard } \\
s \text { hard } \\
s \text { hard } \\
s \text { hard } \\
s \text { hard }\end{array}$ & $\begin{array}{l}\text { Friable } \\
\text { friable } \\
\text { friable } \\
\text { friable } \\
\text { friable }\end{array}$ & $\begin{array}{c}\text { gradual s } \\
\text { diffuse } \\
\text { gradual s } \\
\text { gradual s } \\
-\end{array}$ \\
\hline & & 15 & +55 & $\begin{array}{c}0-30 \\
30-80 \\
80-120 \\
\end{array}$ & $\begin{array}{l}\text { 10YR 6/4 } \\
7.5 Y R \text { 5/6 } \\
7.5 Y R \text { 6/6 } \\
\end{array}$ & $\begin{array}{l}5 / 4 \\
4 / 6 \\
5 / 6 \\
\end{array}$ & $\begin{array}{l}2 \mathrm{c} \text { to } \mathrm{m} \mathrm{sbk} \\
1 \mathrm{~m} \text { to } \mathrm{f} \mathrm{sbk} \\
1 \mathrm{~m} \text { to } \mathrm{f} \mathrm{sbk}\end{array}$ & $\begin{array}{l}\text { ex hard } \\
\text { s hard } \\
\text { s hard }\end{array}$ & $\begin{array}{l}\text { friable } \\
\text { friable } \\
\text { friable } \\
\end{array}$ & $\begin{array}{c}\text { Abrupt } \\
\text { gradual s } \\
-\end{array}$ \\
\hline & 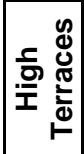 & 16 & +67 & $\begin{array}{c}0-40 \\
40-75 \\
75-90 \\
90-135\end{array}$ & $\begin{array}{l}\text { 10YR 6/4 } \\
\text { 10YR 7/4 } \\
\text { 10YR 6/6 } \\
\text { 10YR 6/6 }\end{array}$ & $\begin{array}{l}5 / 4 \\
6 / 4 \\
5 / 6 \\
5 / 6\end{array}$ & $\begin{array}{c}1 \mathrm{~m} \text { to } f \text { sbk } \\
\mathrm{sg} \\
1 \mathrm{~m} \text { to } f \mathrm{sbk} \\
\mathrm{sg}\end{array}$ & $\begin{array}{c}\text { soft } \\
\text { loose } \\
\text { soft } \\
\text { loose }\end{array}$ & $\begin{array}{l}\text { friable } \\
\text { loose } \\
\text { friable } \\
\text { loose }\end{array}$ & $\begin{array}{c}\text { abrupt } \\
\text { gradual s } \\
\text { abrupt } \\
-\end{array}$ \\
\hline & \multirow{3}{*}{ 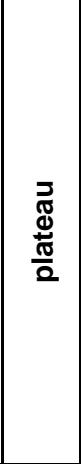 } & 17 & +72 & $\begin{array}{c}0-60 \\
60-90 \\
90-120 \\
120-150\end{array}$ & $\begin{array}{l}\text { 10YR 6/6 } \\
\text { 10YR 6/6 } \\
\text { 10YR 6/6 } \\
\text { 10YR 7/6 }\end{array}$ & $\begin{array}{l}5 / 6 \\
5 / 6 \\
5 / 6 \\
6 / 6\end{array}$ & $\begin{array}{c}1 \mathrm{fg}+\mathrm{sg} \\
\mathrm{sg} \\
\mathrm{sg} \\
\mathrm{sg}\end{array}$ & $\begin{array}{l}\text { loose } \\
\text { loose } \\
\text { loose } \\
\text { loose }\end{array}$ & $\begin{array}{l}\text { Loose } \\
\text { loose } \\
\text { loose } \\
\text { loose }\end{array}$ & $\begin{array}{l}\text { diffuse } \\
\text { diffuse } \\
\text { diffuse } \\
-\end{array}$ \\
\hline & & 18 & +80 & $\begin{array}{c}0-38 \\
38-77 \\
\end{array}$ & $\begin{array}{l}\text { 10YR 6/6 } \\
\text { 10YR 6/6 } \\
\end{array}$ & $\begin{array}{l}5 / 6 \\
4 / 6 \\
\end{array}$ & $\begin{array}{c}1 \mathrm{f} \text { sbk to } g r \\
1 \mathrm{f} \mathrm{sbk}\end{array}$ & $\begin{array}{l}\text { s hard } \\
\text { s hard }\end{array}$ & $\begin{array}{l}\text { friable } \\
\text { friable }\end{array}$ & $\begin{array}{c}\text { clear } \\
- \\
\end{array}$ \\
\hline & & 19 & +84 & $\begin{array}{c}0-25 \\
25-50 \\
50-70 \\
70-90 \\
90-110 \\
110-140\end{array}$ & $\begin{array}{c}7.5 \text { YR } 6 / 6 \\
10 Y \text { R } 6 / 6 \\
10 \text { YR } 7 / 5 \\
7.5 \text { YR } 6 / 6 \\
7.5 \text { YR } 6 / 6 \\
7.5 Y R 6 / 6\end{array}$ & $\begin{array}{l}5 / 6 \\
5 / 6 \\
6 / 5 \\
5 / 6 \\
5 / 6 \\
5 / 6\end{array}$ & $\begin{array}{l}\text { sg } \\
\text { sg } \\
\text { sg } \\
\text { sg } \\
\text { sg } \\
\text { sg }\end{array}$ & $\begin{array}{l}\text { loose } \\
\text { loose } \\
\text { loose } \\
\text { loose } \\
\text { loose } \\
\text { loose }\end{array}$ & $\begin{array}{l}\text { Loose } \\
\text { loose } \\
\text { loose } \\
\text { loose } \\
\text { loose } \\
\text { loose }\end{array}$ & $\begin{array}{c}\text { clear } \\
\text { gradual s } \\
\text { clear } \\
\text { diffuse } \\
\text { diffuse } \\
-\end{array}$ \\
\hline
\end{tabular}

Abbreviations: Texture*: L=loamy, $\mathrm{S}=$ sandy, s g=slightly gravely, g=gravely; Structure ${ }^{1}:$ 1=weak, 2 = moderate, $\mathrm{v}=$ very, $\mathrm{f}=$ fine, $\mathrm{m}=$ medium, $\mathrm{c}=$ coarse, $\mathrm{gr}=$ granular, $\mathrm{sbk}=$ subangular blocky, $\mathrm{bk}=$ blocky, $\mathrm{sg}=$ single grains; Consistence ${ }^{2}: \mathrm{v}=$ very, $\mathrm{x}=$ extremely; Boundary ${ }^{3}: \mathrm{s}=$ smooth

\section{Physiochemical properties}

The physiochemical properties of the studied soils are registered in Table (3). Data in Table (3) show that, most of the studied soils have slightly to highly gravely sandy, loamy sand to sandy loam texture. Only the soils of low alluvial plain terraces have clay loam texture (as w.p.m.). Most of the studied soils are nonsaline (EC $<2 \mathrm{dSm}^{-1}$, as w.p.m.), haven't sodicity effect and having slightly to moderately alkaline reaction $(\mathrm{pH}>7$ to $<$
8.5 as w.p.m.). Only soils of profile 19 in the High Aeoline Terraces had shown a clear salinity $\left(E C>4 \mathrm{dSm}^{-1}\right)$ and sodicity (> $15 \%$ ESP) effects.

These soils are slightly to moderately calcareous having $<7 \% \mathrm{CaCO}_{3}$ content (as w.p.m.). Gypsum content is low (<3\%). Organic matter $(O M)$ is low $(<2 \%$, as w.p.m.). The cation exchange capacity (CEC) is depending on the fine fractions and organic matter contents. 
M.S. Amira, et al.,

Table (3): Some physical and chemical properties of studied soil profiles.

\begin{tabular}{|c|c|c|c|c|c|c|c|c|c|c|c|c|c|c|c|}
\hline \multirow{2}{*}{ 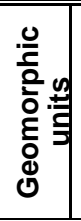 } & \multirow{2}{*}{ 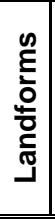 } & \multirow{2}{*}{ 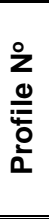 } & \multirow{2}{*}{$\begin{array}{l}\text { Depth } \\
\text { Cm }\end{array}$} & \multirow{2}{*}{$\begin{array}{l}\text { d0 } \\
\frac{0}{0} \\
\sum_{0}^{\frac{\pi}{0}} \\
\frac{0}{0}\end{array}$} & \multicolumn{3}{|c|}{$\begin{array}{c}\text { Particle size } \\
\text { distribution \% }\end{array}$} & \multirow{2}{*}{ 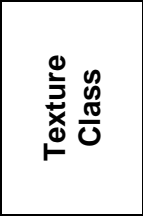 } & \multirow{2}{*}{ 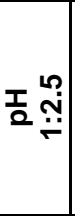 } & \multirow{2}{*}{ 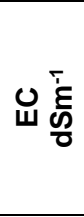 } & \multirow{2}{*}{ 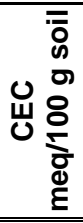 } & \multirow[b]{2}{*}{ ESP } & \multirow{2}{*}{ 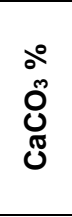 } & \multirow{2}{*}{ 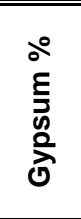 } & \multirow{2}{*}{$\begin{array}{c}\text { OM } \\
\%\end{array}$} \\
\hline & & & & & ஸ્ & $\overline{\bar{\omega}}$ & $\frac{\widehat{\pi}}{U}$ & & & & & & & & \\
\hline \multirow{6}{*}{ 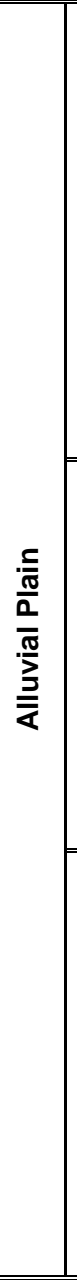 } & \multirow{2}{*}{ 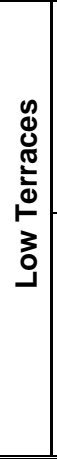 } & 1 & $\begin{array}{c}0-30 \\
30-60 \\
60-90 \\
90-110 \\
110-130 \\
\text { W.P.M }\end{array}$ & $\begin{array}{l}- \\
- \\
- \\
- \\
- \\
-\end{array}$ & \begin{tabular}{|l|}
38.1 \\
36.1 \\
34.3 \\
34.2 \\
34.6 \\
35.6
\end{tabular} & $\begin{array}{l}32.6 \\
33.3 \\
33.5 \\
33.6 \\
34.0 \\
33.3\end{array}$ & \begin{tabular}{l|}
29.3 \\
30.6 \\
32.2 \\
32.2 \\
31.4 \\
30.1
\end{tabular} & $\begin{array}{l}\text { Clay L. } \\
\text { Clay L. } \\
\text { Clay L. } \\
\text { Clay L. } \\
\text { Clay L. } \\
\text { Clay L. }\end{array}$ & $\begin{array}{l}7.5 \\
7.5 \\
7.6 \\
7.9 \\
8.1 \\
7.7 \\
\end{array}$ & $\begin{array}{l}0.39 \\
0.27 \\
0.26 \\
0.24 \\
0.22 \\
0.28 \\
\end{array}$ & $\begin{array}{l}40.6 \\
28.6 \\
26.5 \\
26.5 \\
24.4 \\
29.9\end{array}$ & $\begin{array}{l}3.22 \\
4.08 \\
4.22 \\
5.25 \\
3.87 \\
4.06 \\
\end{array}$ & $\begin{array}{l}1.59 \\
1.81 \\
2.27 \\
1.31 \\
0.23 \\
1.55 \\
\end{array}$ & \begin{tabular}{c|}
1.1 \\
0.9 \\
0.8 \\
1.1 \\
1.3 \\
1.02 \\
\end{tabular} & $\begin{array}{l}0.84 \\
0.69 \\
0.34 \\
0.22 \\
0.08 \\
0.48\end{array}$ \\
\hline & & 2 & $\begin{array}{c}0-30 \\
30-55 \\
55-70 \\
70-100 \\
100-130 \\
130-150 \\
\text { W.P.M }\end{array}$ & $\begin{array}{l}- \\
- \\
- \\
- \\
- \\
- \\
-\end{array}$ & \begin{tabular}{l|}
41.2 \\
37.8 \\
36.9 \\
36.1 \\
33.5 \\
32.0 \\
36.3 \\
\end{tabular} & $\begin{array}{l}30.6 \\
31.8 \\
32.4 \\
32.8 \\
34.2 \\
35.0 \\
32.7 \\
\end{array}$ & \begin{tabular}{l|}
28.2 \\
30.4 \\
30.7 \\
31.6 \\
32.3 \\
33.0 \\
31.0
\end{tabular} & $\begin{array}{l}\text { Clay L. } \\
\text { Clay L. } \\
\text { Clay L. } \\
\text { Clay L. } \\
\text { Clay L. } \\
\text { Clay L. } \\
\text { Clay L. }\end{array}$ & $\begin{array}{l}7.7 \\
7.7 \\
7.7 \\
7.8 \\
7.8 \\
7.8 \\
7.8 \\
\end{array}$ & $\begin{array}{l}0.25 \\
0.24 \\
0.23 \\
0.21 \\
0.21 \\
0.21 \\
0.23 \\
\end{array}$ & $\begin{array}{l}35.5 \\
32.2 \\
35.0 \\
28.7 \\
22.4 \\
21.0 \\
29.0 \\
\end{array}$ & \begin{tabular}{l|}
2.59 \\
1.92 \\
2.77 \\
2.83 \\
3.38 \\
3.48 \\
2.82 \\
\end{tabular} & $\begin{array}{l}0.54 \\
1.18 \\
0.91 \\
1.54 \\
1.90 \\
1.54 \\
1.29 \\
\end{array}$ & \begin{tabular}{c|}
1.5 \\
1.1 \\
0.9 \\
0.8 \\
0.7 \\
0.6 \\
0.95 \\
\end{tabular} & $\begin{array}{l}0.55 \\
0.18 \\
0.15 \\
0.25 \\
0.25 \\
0.50 \\
0.32\end{array}$ \\
\hline & \multirow{2}{*}{ 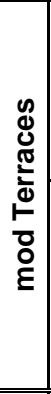 } & 3 & $\begin{array}{c}0-30 \\
30-60 \\
60-90 \\
90-140 \\
\text { W.P.M }\end{array}$ & $\begin{array}{l}40.0 \\
18.0 \\
38.0 \\
63.6 \\
43.3\end{array}$ & $\begin{array}{l}85.9 \\
86.8 \\
87.8 \\
88.5 \\
87.4\end{array}$ & $\begin{array}{c}10.2 \\
10.0 \\
9.5 \\
9.2 \\
9,7\end{array}$ & $\begin{array}{l}3.9 \\
3.2 \\
2.7 \\
2.3 \\
2.9\end{array}$ & $\begin{array}{c}\text { Loamy S. } \\
\text { Sand } \\
\text { Sand } \\
\text { Sand } \\
\text { Sand }\end{array}$ & $\begin{array}{l}7.1 \\
7.3 \\
7.1 \\
7.7 \\
7.3\end{array}$ & $\begin{array}{l}1.53 \\
0.61 \\
0.20 \\
0.19 \\
0.57\end{array}$ & $\begin{array}{c}10.5 \\
9.1 \\
8.4 \\
8.4 \\
9.0\end{array}$ & $\begin{array}{l}10.19 \\
8.33 \\
9.67 \\
8.70 \\
9.15\end{array}$ & $\begin{array}{l}1.77 \\
0.59 \\
1.09 \\
0.95 \\
1.08\end{array}$ & $\begin{array}{c}1.5 \\
1.9 \\
2.0 \\
1.7 \\
1.76\end{array}$ & $\begin{array}{l}0.29 \\
0.20 \\
0.17 \\
0.17 \\
0.20\end{array}$ \\
\hline & & 4 & $\begin{array}{c}0-30 \\
30-60 \\
60-90 \\
90-120 \\
120-150 \\
\text { W.P.M } \\
\end{array}$ & \begin{tabular}{c|}
25.0 \\
33.6 \\
14.9 \\
8.6 \\
6.3 \\
17.7 \\
\end{tabular} & \begin{tabular}{|l|}
83.5 \\
84.5 \\
85.3 \\
86.0 \\
86.5 \\
85.2 \\
\end{tabular} & $\begin{array}{l}11.6 \\
11.0 \\
10.9 \\
10.4 \\
10.3 \\
10.8 \\
\end{array}$ & \begin{tabular}{l|}
4.9 \\
4.5 \\
3.8 \\
3.6 \\
3.2 \\
4.0 \\
\end{tabular} & $\begin{array}{l}\text { Loamy S. } \\
\text { Loamy S. } \\
\text { Loamy S. } \\
\text { Loamy S. } \\
\text { Loamy S. } \\
\text { Loamy S. }\end{array}$ & $\begin{array}{l}7.9 \\
7.8 \\
7.9 \\
8.1 \\
8.1 \\
8.0 \\
\end{array}$ & \begin{tabular}{l|l|}
0.23 \\
0.25 \\
0.18 \\
0.18 \\
0.17 \\
0.20 \\
\end{tabular} & \begin{tabular}{l|}
12.6 \\
11.9 \\
10.5 \\
10.5 \\
10.5 \\
11.2 \\
\end{tabular} & \begin{tabular}{l|}
5.58 \\
5.44 \\
5.34 \\
3.65 \\
3.94 \\
4.76 \\
\end{tabular} & $\begin{array}{l}1.31 \\
2.58 \\
1.59 \\
0.63 \\
0.41 \\
1.30 \\
\end{array}$ & $\begin{array}{l}1.1 \\
1.5 \\
1.8 \\
1.9 \\
1.2 \\
1.5 \\
\end{array}$ & $\begin{array}{l}0.39 \\
0.20 \\
0.20 \\
0.17 \\
0.10 \\
0.21 \\
\end{array}$ \\
\hline & \multirow{2}{*}{ 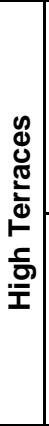 } & 5 & $\begin{array}{c}0-30 \\
30-60 \\
60-90 \\
90-120 \\
120-150 \\
\text { W.P.M } \\
\end{array}$ & \begin{tabular}{l|l|}
16.7 \\
17.1 \\
17.3 \\
18.4 \\
16.3 \\
17.2 \\
\end{tabular} & \begin{tabular}{l|}
85.1 \\
85.7 \\
86.4 \\
87.0 \\
87.7 \\
86.4 \\
\end{tabular} & $\begin{array}{c}10.6 \\
10.1 \\
9.9 \\
9.5 \\
9.1 \\
9.8 \\
\end{array}$ & \begin{tabular}{l|}
4.3 \\
4.2 \\
3.7 \\
3.5 \\
3.1 \\
3.8 \\
\end{tabular} & $\begin{array}{l}\text { Sand } \\
\text { Sand } \\
\text { Sand } \\
\text { Sand } \\
\text { Sand } \\
\text { Sand } \\
\end{array}$ & $\begin{array}{l}7.2 \\
7.3 \\
7.5 \\
7.5 \\
7.5 \\
7.4 \\
\end{array}$ & \begin{tabular}{l|}
0.13 \\
0.38 \\
0.39 \\
0.51 \\
0.58 \\
0.43 \\
\end{tabular} & \begin{tabular}{|c|}
15.5 \\
14.5 \\
14.2 \\
13.1 \\
8.8 \\
13.2 \\
\end{tabular} & & $\begin{array}{l}0.50 \\
0.45 \\
0.72 \\
0.86 \\
0.77 \\
0.66 \\
\end{array}$ & $\begin{array}{l}1.7 \\
1.5 \\
2.1 \\
2.3 \\
2.6 \\
2.0 \\
\end{array}$ & $\begin{array}{l}0.10 \\
0.12 \\
0.02 \\
0.12 \\
0.12 \\
0.09\end{array}$ \\
\hline & & 6 & $\begin{array}{c}0-30 \\
30-60 \\
60-90 \\
90-120 \\
120-150 \\
\text { W.P.M } \\
\end{array}$ & \begin{tabular}{l|}
23.8 \\
22.8 \\
22.2 \\
19.6 \\
18.5 \\
21.4 \\
\end{tabular} & $\begin{array}{l}84.7 \\
82.8 \\
83.3 \\
82.0 \\
80.3 \\
82.6 \\
\end{array}$ & $\begin{array}{l}12.0 \\
12.5 \\
12.5 \\
12.7 \\
13.2 \\
12.6 \\
\end{array}$ & $\begin{array}{l}3.3 \\
4.7 \\
4.2 \\
5.3 \\
6.5 \\
4.8 \\
\end{array}$ & $\begin{array}{l}\text { Loamy S. } \\
\text { Loamy S. } \\
\text { Loamy S. } \\
\text { Loamy S. } \\
\text { Loamy S. } \\
\text { Loamy S. }\end{array}$ & $\begin{array}{l}7.5 \\
7.6 \\
8.0 \\
8.6 \\
8.9 \\
8.1 \\
\end{array}$ & $\begin{array}{l}0.36 \\
0.19 \\
0.16 \\
0.15 \\
0.21 \\
0.21 \\
\end{array}$ & $\begin{array}{l}9.2 \\
8.5 \\
6.7 \\
6.7 \\
5.8 \\
7.4 \\
\end{array}$ & $\begin{array}{l}7.02 \\
6.61 \\
7.51 \\
6.18 \\
6.59 \\
6.78 \\
\end{array}$ & $\begin{array}{l}1.90 \\
3.17 \\
2.49 \\
2.22 \\
1.77 \\
2.31 \\
\end{array}$ & $\begin{array}{l}2.5 \\
1.3 \\
1.8 \\
1.1 \\
1.9 \\
1.7 \\
\end{array}$ & $\begin{array}{l}0.34 \\
0.24 \\
0.18 \\
0.15 \\
0.13 \\
0.21 \\
\end{array}$ \\
\hline \multirow{3}{*}{$\begin{array}{l}\frac{5}{\frac{\pi}{0}} \\
\frac{2}{0} \\
\frac{0}{0}\end{array}$} & \multirow{3}{*}{ 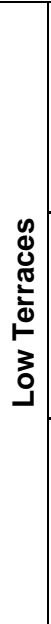 } & 7 & $\begin{array}{c}0-30 \\
30-60 \\
60-90 \\
90-120 \\
120-150 \\
\text { W.P.M }\end{array}$ & \begin{tabular}{l|}
34.3 \\
37.4 \\
30.6 \\
28.8 \\
26.7 \\
31.6
\end{tabular} & \begin{tabular}{|l|}
83.1 \\
80.9 \\
81.6 \\
79.3 \\
75.1 \\
78.0
\end{tabular} & $\begin{array}{l}12.3 \\
13.6 \\
13.2 \\
14.0 \\
17.6 \\
14.1\end{array}$ & $\begin{array}{l}4.6 \\
5.5 \\
5.2 \\
6.7 \\
7.3 \\
5.9\end{array}$ & $\begin{array}{l}\text { Loamy S. } \\
\text { Loamy S. } \\
\text { Loamy S. } \\
\text { Loamy S. } \\
\text { Sandy L. } \\
\text { Loamy S. }\end{array}$ & $\begin{array}{l}7.6 \\
7.8 \\
7.8 \\
7.9 \\
8.2 \\
7.9 \\
\end{array}$ & $\begin{array}{l}1.52 \\
0.59 \\
0.41 \\
0.23 \\
0.19 \\
0.59\end{array}$ & \begin{tabular}{c|}
16.2 \\
14.7 \\
14.0 \\
10.5 \\
9.7 \\
13.0
\end{tabular} & $\begin{array}{l}4.00 \\
5.16 \\
6.18 \\
4.79 \\
5.16 \\
5.06\end{array}$ & $\begin{array}{l}3.99 \\
6.66 \\
5.17 \\
4.53 \\
4.94 \\
5.06\end{array}$ & $\begin{array}{l}1.7 \\
1.5 \\
1.3 \\
0.9 \\
1.7 \\
1.4\end{array}$ & $\begin{array}{l}0.30 \\
0.25 \\
0.25 \\
0.17 \\
0.17 \\
0.23\end{array}$ \\
\hline & & 8 & $\begin{array}{c}0-30 \\
30-60 \\
60-90 \\
90-120 \\
120-150 \\
\text { W.P.M }\end{array}$ & \begin{tabular}{l|}
30.0 \\
16.4 \\
24.6 \\
20.2 \\
14.7 \\
20.6 \\
\end{tabular} & \begin{tabular}{|l|}
76.3 \\
75.7 \\
83.4 \\
78.8 \\
78.2 \\
78.8 \\
\end{tabular} & $\begin{array}{l}19.6 \\
19.8 \\
11.7 \\
14.7 \\
12.5 \\
14.9 \\
\end{array}$ & $\begin{array}{l}4.1 \\
4.5 \\
4.9 \\
6.5 \\
9.3 \\
6.3 \\
\end{array}$ & $\begin{array}{l}\text { sandy L } \\
\text { Sandy L. } \\
\text { Loamy S. } \\
\text { Loamy S. } \\
\text { Loamy S. } \\
\text { Loamy S. }\end{array}$ & $\begin{array}{l}7.9 \\
7.5 \\
7.5 \\
7.2 \\
7.3 \\
7.5 \\
\end{array}$ & $\begin{array}{l}0.19 \\
0.43 \\
0.33 \\
0.30 \\
0.22 \\
0.28 \\
\end{array}$ & \begin{tabular}{l|}
23.8 \\
39.2 \\
14.7 \\
16.8 \\
21.0 \\
21.8 \\
\end{tabular} & $\begin{array}{l}2.84 \\
3.15 \\
6.60 \\
5.15 \\
1.68 \\
3.77 \\
\end{array}$ & $\begin{array}{l}7.11 \\
2.95 \\
6.57 \\
4.71 \\
3.22 \\
4.83 \\
\end{array}$ & \begin{tabular}{l|}
1.5 \\
1.2 \\
2.3 \\
2.5 \\
1.6 \\
1.8 \\
\end{tabular} & $\begin{array}{l}0.37 \\
1.38 \\
0.76 \\
0.17 \\
0.17 \\
0.49 \\
\end{array}$ \\
\hline & & 9 & $\begin{array}{c}0-30 \\
30-60 \\
60-90 \\
90-120 \\
120-150 \\
\text { W.P.M }\end{array}$ & \begin{tabular}{l|}
24.2 \\
27.2 \\
28.4 \\
50.4 \\
38.1 \\
33.7
\end{tabular} & \begin{tabular}{l|}
83.5 \\
81.8 \\
79.1 \\
79.3 \\
76.5 \\
80.0
\end{tabular} & $\begin{array}{l}12.3 \\
13.4 \\
15.0 \\
14.5 \\
16.3 \\
14.3\end{array}$ & $\begin{array}{l}4.2 \\
4.8 \\
5.9 \\
6.2 \\
7.2 \\
5.7\end{array}$ & \begin{tabular}{|l|} 
Loamy S. \\
Loamy S. \\
Loamy S. \\
Loamy S. \\
Loamy L.
\end{tabular} & $\begin{array}{l}7.1 \\
7.3 \\
7.5 \\
7.8 \\
7.8 \\
7.5 \\
\end{array}$ & $\begin{array}{l}0.53 \\
0.37 \\
0.19 \\
0.17 \\
0.16 \\
0.28\end{array}$ & \begin{tabular}{l|}
23.1 \\
21.7 \\
14.0 \\
13.7 \\
10.2 \\
16.6
\end{tabular} & $\begin{array}{l}4.63 \\
4.23 \\
4.82 \\
4.51 \\
6.34 \\
4.91 \\
\end{array}$ & \begin{tabular}{l|}
2.76 \\
2.72 \\
1.54 \\
1.81 \\
1.77 \\
2.12
\end{tabular} & $\begin{array}{l}1.5 \\
2.1 \\
2.3 \\
2.4 \\
2.8 \\
2.2\end{array}$ & $\begin{array}{l}0.49 \\
0.25 \\
0.18 \\
0.17 \\
0.13 \\
0.25\end{array}$ \\
\hline
\end{tabular}

W.P.M = weighted profile means, L.= loam, S.= Sand 
Table (3): Cont.

\begin{tabular}{|c|c|c|c|c|c|c|c|c|c|c|c|c|c|c|c|}
\hline \multirow{2}{*}{ 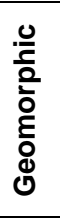 } & \multirow{2}{*}{ 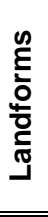 } & \multirow{2}{*}{$\begin{array}{l}\frac{0}{Z} \\
\frac{0}{n} \\
\frac{0}{2} \\
\frac{0}{\alpha}\end{array}$} & \multirow{2}{*}{$\begin{array}{l}\text { Depth } \\
\text { cm }\end{array}$} & \multirow{2}{*}{$\begin{array}{l}\frac{0}{0} \\
0 \\
0 \\
\frac{\pi}{0} \\
0\end{array}$} & \multicolumn{3}{|c|}{$\begin{array}{c}\text { Particle size } \\
\text { distribution } \%\end{array}$} & \multirow{2}{*}{ 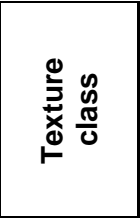 } & \multirow{2}{*}{ 돌 $\stackrel{10}{\stackrel{n}{n}}$} & \multirow{2}{*}{ ن } & \multirow{2}{*}{ 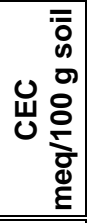 } & \multirow{2}{*}{$\begin{array}{l}\text { ○ } \\
\text { 崩 }\end{array}$} & \multirow{2}{*}{ 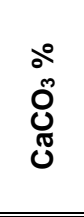 } & \multirow{2}{*}{$\begin{array}{l}\delta^{0} \\
\sum_{0}^{5} \\
\sum_{0}^{0} \\
0\end{array}$} & \multirow{2}{*}{$\sum_{0}^{0}$} \\
\hline & & & & & ஸ̆ & 岛 & $\frac{\widehat{\pi}}{U}$ & & & & & & & & \\
\hline & & & $0-50$ & & 80.4 & 14.2 & 5.4 & Loamy S. & 6.9 & 0.57 & 14.0 & 8.16 & 5.75 & 2.5 & 0.40 \\
\hline & & & $50-70$ & 44.1 & 76.2 & 17.3 & 6.5 & Sandy L. & 8.1 & 0.26 & 15.4 & 4.56 & 7.48 & 1.9 & 0.47 \\
\hline & & 10 & $70-110$ & 34.4 & 74.1 & 18.9 & 7.0 & Sandy L. & 8.0 & 0.28 & 12.0 & 9.31 & 7.20 & 2.3 & 0.44 \\
\hline & & & $110-150$ & 85.0 & 73.7 & 18.8 & 7.5 & Sandy L. & 8.1 & 0.24 & 10.8 & 7.02 & 3.22 & 2.0 & 0.22 \\
\hline & & & W.P.M & 52.1 & 76.4 & 17.1 & 6.5 & Sandy L. & 7.8 & 0.37 & 12.8 & 7.68 & 5.69 & 2.2 & 0.37 \\
\hline & & & $0-30$ & 29.2 & 74.1 & 17.6 & 8.3 & Sandy L. & 7.9 & 0.46 & 20.3 & 3.73 & 4.21 & 1.3 & 1.32 \\
\hline & ల్ర & & $30-60$ & 31.5 & 75.5 & 17.3 & 7.2 & Sandy L. & 7.9 & 0.39 & 18.9 & 6.78 & 2.76 & 1.2 & 0.65 \\
\hline$\frac{10}{a}$ & $\frac{2}{d}$ & & $60-90$ & 28.5 & 75.1 & 18.9 & 6.0 & Sandy L. & 8.2 & 0.27 & 17.5 & 4.95 & 3.94 & 1.1 & 0.40 \\
\hline 후 & 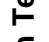 & 11 & $90-120$ & 24.0 & 74.7 & 19.1 & 6.2 & Sandy L. & 7.6 & 0.18 & 17.5 & 2.02 & 2.72 & 1.2 & 0.30 \\
\hline ه & 동 & & $120-123$ & 33.0 & 75.5 & 19.3 & 5.2 & Sandy L. & 7.9 & 0.17 & 17.5 & 3.37 & 1.84 & 1.4 & 0.10 \\
\hline & & & W.P.M & 28.4 & 74.9 & 18.2 & 6.9 & Sandy L. & 7.9 & 0.32 & 18.5 & 4.35 & 3.77 & 1.2 & 0.66 \\
\hline & & & $0-20$ & 46.7 & 78.6 & 15.2 & 6.2 & Loamy S. & 8.2 & 0.25 & 10.5 & 5.07 & 4.58 & 1.1 & 0.76 \\
\hline & & & $20-70$ & 58. & 74.8 & 18.4 & 6.8 & Iy L. & 8.1 & 0.18 & 11.2 & 3.96 & 10.06 & 1.3 & 0.42 \\
\hline & & 12 & $70-100$ & 49.6 & 72.2 & 20.3 & 7.5 & Sandy L. & 7.7 & 0.15 & 14.0 & 4.62 & 8.61 & 1.3 & 0.26 \\
\hline & & & $100-140$ & 32.0 & 70.5 & 21.2 & 8.3 & Sandy L. & 7.4 & 0.15 & 14.7 & 3.02 & 1.90 & 1.4 & 0.17 \\
\hline & & & W.P.M & 47.2 & 73.5 & 19.2 & 7.3 & Sandy L. & 7.8 & 0.18 & 12.7 & 3.99 & 6.64 & 1.3 & 0.36 \\
\hline & & & $0-20$ & 17.4 & 81.0 & 14.2 & 4.8 & Loamy S. & 7.5 & 0.68 & 14.0 & 9.48 & 1.59 & 1.4 & 0.24 \\
\hline & & & $20-40$ & 14. & 83.2 & 12.5 & 4.3 & Loamy S. & 8.1 & 0.21 & 14.0 & 4.82 & 3.35 & 1.3 & 0.17 \\
\hline & & & $40-100$ & 65.9 & 84.9 & 10.5 & 4.6 & Loamy S. & 8.2 & 0.21 & 14.7 & 4.78 & 8.65 & 0.9 & 0.15 \\
\hline & & $\mathbf{I}$ & $100-110$ & 72.5 & 85.4 & 10.4 & 4.2 & Loamy S. & 8.2 & 0.20 & 13.4 & 5.25 & 5.44 & 1.4 & 0.12 \\
\hline & & & $110-150$ & 53.3 & 84.9 & 10.6 & 4.5 & Loamy S. & 8.5 & 0.20 & 13.4 & 5.66 & 2.95 & 1.0 & 0.08 \\
\hline & & & W.P.M & 49.7 & 84.2 & 11.3 & 4.5 & Loamy S. & 8.1 & 0.29 & 14.1 & 5.68 & 5.27 & 1.1 & 0.14 \\
\hline & U్ర & & $0-30$ & 20.3 & 84.0 & 12.3 & 3.7 & Loamy S. & 8.2 & 0.44 & 9.1 & 7.11 & 3.67 & 0.8 & 0.15 \\
\hline & $\frac{2}{2}$ & & $30-60$ & 17.5 & 82.9 & 12.2 & 4.9 & Loamy S. & 8.2 & 0.39 & 12.6 & 5.80 & 4.44 & 0.8 & 0.12 \\
\hline & $\stackrel{-}{-1}$ & 11 & $60-90$ & 21.8 & 81.8 & 12.9 & 5.3 & Loamy S. & 8.4 & 0.36 & 12.3 & 3.34 & 4.89 & 0.6 & 0.02 \\
\hline & 3 & 14 & $90-120$ & 29.0 & 80.1 & 13.4 & 6.5 & Loamy S. & 8.5 & 0.32 & 12.5 & 7.76 & 6.52 & 1.0 & 0.08 \\
\hline & & & $120-150$ & $17 . \varepsilon$ & 79.7 & 13.9 & 6.4 & Loamy S. & 8.6 & 0.24 & 12.0 & 5.86 & 4.62 & 1.0 & 0.03 \\
\hline & & & W.P.M & 21.3 & 81.7 & 12.9 & 5.4 & Loamy S. & 8.4 & 0.35 & 11.9 & 5.97 & 4.83 & 0.9 & 0.08 \\
\hline & & & $0-30$ & 28.6 & 82.8 & 12.9 & 4.3 & Loamy S. & 7.2 & 1.39 & 14.0 & 13.56 & 5.35 & 1.1 & 1.01 \\
\hline & & 15 & $30-80$ & 64.3 & 81.8 & 13.3 & 4.9 & Loamy S. & 7.4 & 0.65 & 10.5 & 12.64 & 9.83 & 0.8 & 0.52 \\
\hline & & 10 & $80-120$ & 27.0 & 74.9 & 17.3 & 7.8 & Sandy L. & 7.4 & 0.53 & 8.4 & 14.45 & 3.85 & 1.1 & 0.45 \\
\hline & & & W.P.M & 42.9 & 79.8 & 14.5 & 5.7 & Loamy S. & 7.4 & 0.80 & 10.7 & 13.47 & 6.72 & 1.0 & 0.62 \\
\hline "స⿱一兀 & d & & $0-40$ & 17.0 & 72.7 & 19.0 & 8.3 & Sandy L. & 7.0 & 1.49 & 21.0 & 5.55 & 2.95 & 1.0 & 0.84 \\
\hline & $\stackrel{\pi}{2}$ & & $40-75$ & 2.7 & 74.6 & 18.2 & 7.2 & Sandy L. & 7.8 & 0.29 & 10.5 & 3.94 & 0.23 & 1.0 & 0.76 \\
\hline & 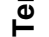 & 16 & $75-90$ & 11.6 & 79.7 & 15.3 & 5.0 & Loamy S. & 7.8 & 0.27 & 14.7 & 5.71 & 1.99 & 1.1 & 0.39 \\
\hline & ¿ & & $90-135$ & 86.7 & 81.9 & 11.2 & 6.9 & Loamy S. & 7.8 & 0.25 & 14.0 & 6.37 & 4.94 & 1.2 & 0.45 \\
\hline & & & W.P.M & 35.9 & 77.0 & 15.8 & 7.2 & Sandy L. & 7.6 & 0.63 & 15.2 & 5.42 & 2.80 & 1.1 & 0.64 \\
\hline & & & $0-60$ & 7.5 & 81.1 & 14.6 & 4.3 & y S. & 7.5 & 0.36 & 10.5 & 10.88 & 0.63 & 1.1 & 0.18 \\
\hline & & & & 12.9 & 79.0 & 15.7 & & $\mathrm{~S}$. & 7.5 & 0.44 & 9.8 & 8.56 & 0.23 & 1.2 & 0.45 \\
\hline & & 17 & $90-120$ & 2.2 & 77.7 & 16.7 & 5.6 & Loamy S. & 8.5 & 0.26 & 9.1 & 6.48 & 0.02 & 1.0 & 0.44 \\
\hline & & & $120-150$ & 6.7 & 77.3 & 17.2 & 5.5 & Loamy S. & 8.5 & 0.12 & 8.5 & 5.22 & 0.09 & 0.8 & 3.36 \\
\hline & & & W.P.M & 7.2 & 79.2 & 15.8 & 5.0 & Loamy S. & 8.0 & 0.41 & 9.7 & 8.41 & 0.32 & 1.1 & 0.92 \\
\hline & ذِ & & $0-38$ & 14.7 & 77.0 & 15.6 & 7.4 & Sandy L. & 7.5 & 1.45 & 28.0 & 5.27 & 6.16 & 1.3 & 1.21 \\
\hline & & 18 & $38-77$ & 25 & 83.1 & 12.3 & 4.6 & Loamy S. & 8.2 & 0.45 & 25.2 & 4.43 & 5.21 & 1.3 & 0.59 \\
\hline & 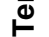 & & W.P.M & 20.2 & 80.1 & 13.9 & 6.0 & y $\mathrm{s}$. & 7.9 & 0.94 & 26.6 & 4.85 & 5.68 & 1.3 & 0.89 \\
\hline & $\overline{0}$ & & $0-25$ & 34.3 & 82.0 & 13.5 & 4.5 & Loa & 7.5 & 10.89 & 10.8 & 57.23 & 3.85 & 1.3 & 0.27 \\
\hline & & & & 40.0 & 82. & 13.5 & 3. & y S. & 7.8 & 6,15 & 11.2 & 26.05 & 7.93 & 1.3 & 0.44 \\
\hline & & & $50-70$ & 6.0 & 80.7 & 13.6 & 5.7 & Loan & 7.9 & 1.24 & 11.5 & 7.98 & 1.72 & 1.1 & 0.35 \\
\hline & & 19 & $70-90$ & 37.6 & 80.4 & 14.2 & 5.4 & Loamy S. & 8.1 & 5.08 & 12.7 & 13.15 & 3.26 & 1.4 & 0.34 \\
\hline & & & $90-110$ & 19. & 78.4 & 15.0 & 6.6 & Loan & 8.2 & 3.59 & 13.1 & 10.63 & 2.49 & 1.3 & 0.02 \\
\hline & & & $110-14$ & 41 & 77.8 & 15.3 & 6.9 & ny $\mathrm{S}$. & 8.4 & 1.59 & 14.0 & 5.80 & 0.68 & 1.3 & 0.15 \\
\hline & & & W.P.M & 31.1 & 80.3 & 14.2 & 5.5 & Loamy S. & 8.0 & 4.80 & 12.3 & 20.65 & 3.32 & 1.3 & 0.26 \\
\hline
\end{tabular}


M.S. Amira, et al.,

\section{Soil classification}

Based on climatic condition, soil morphological, physiochemical characteristics the studied soils are classified up to sub great group level according to Soil Survey Staff (2014). According to FAO (1977) and USDA-NRCS (1997), the dominant soil moisture regime of this area is "Torric" with "Thermic" soil temperature regime. Most of studied soils haven't any diagnostic sub-surface horizons. Therefore, these soils were classified under Entisols Order as Typic Torripsamments or Typic Torriorthents according to their predominant texture grade as presented and showed in Table (4) and Fig (3). Only soils of profile (19) showed the features for occurrence of sodic horizon with ESP > 15\% within 100 $\mathrm{cm}$ of the soil surface. Accordingly, they affiliated to Aridisols as Sodic Haplocambids (Table, 4 and Fig, 3).

Table (4): Soil classification in the study area.

\begin{tabular}{|c|c|c|c|c|}
\hline \multirow{2}{*}{ Order } & \multirow{2}{*}{ Sub Great Group } & \multirow{2}{*}{ Soils of profiles No. } & \multicolumn{2}{|c|}{ Area } \\
\cline { 3 - 5 } Entisols & Typic Torripsamments & $\begin{array}{c}3,4,5,6,7,8,9, \\
13,14,15,17,18\end{array}$ & 5235.44 & 62.25 \\
\cline { 3 - 5 } & Typic Torriorthents & $1,2,10,11,12,16$ & 30369.38 & 36.10 \\
\hline \multirow{2}{*}{ Aridisols } & Sodic Haplocambids & 19 & 1391.10 & 1.65 \\
\hline \multicolumn{2}{|c}{ Total } & 84117.92 & 100 \\
\hline
\end{tabular}

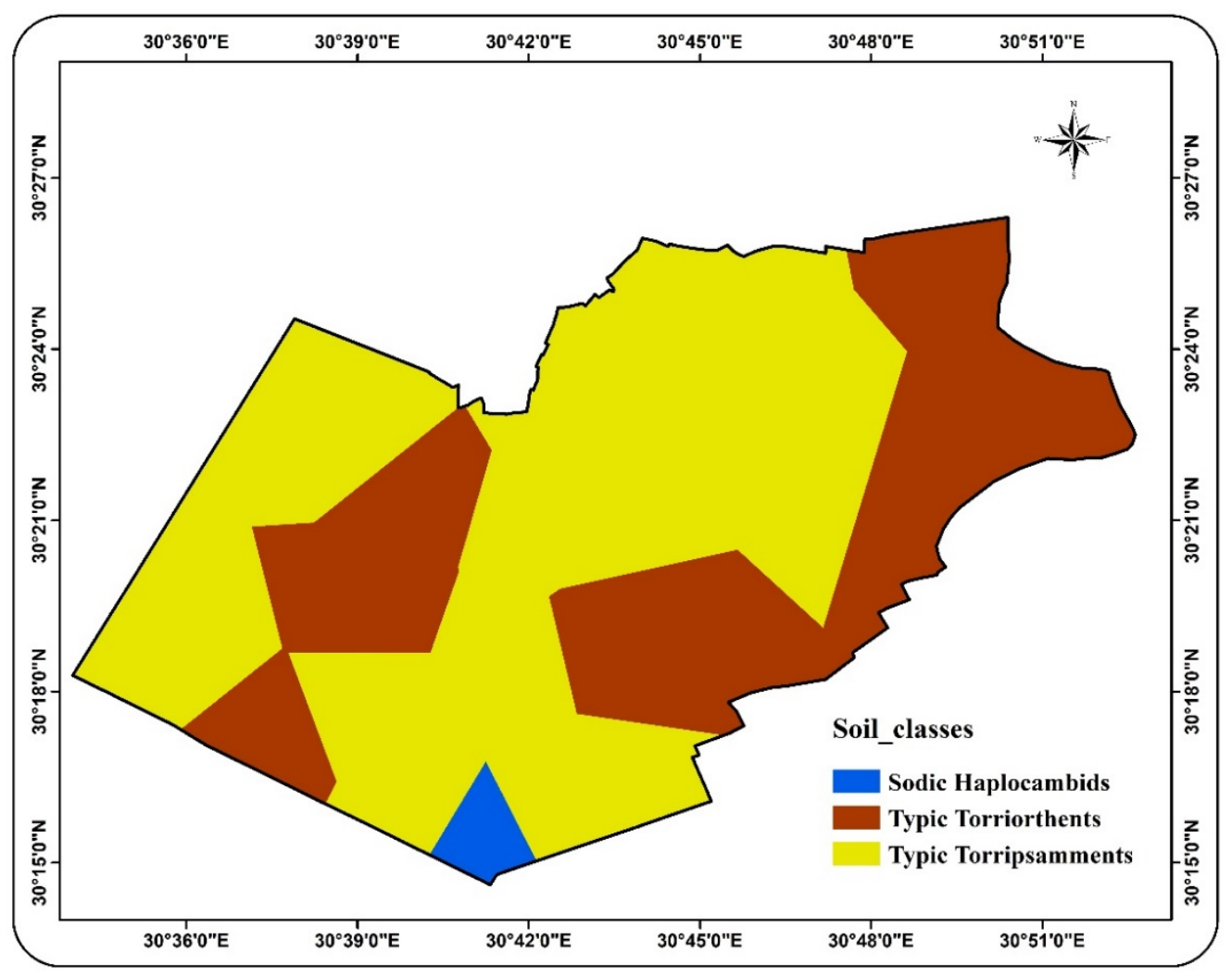

Fig (3): Spatial distribution of soil mapping units in the study area. 


\section{Land capability evaluation}

The Agriculture Land Evaluation System for arid and semi-arid regions (ALES-Arid) model developed by Ismail et al. (1994 and 2005) was used to assess the land capability for the studied soils. The land capability indices for these soils were obtained from the integration between this model and ArcGIS software based on the land, soil, fertility (Table, 5) and irrigation water characteristics (Table,
6). The final land capability indices and classes for the soils of the studied area are presented in Table (7). Also, the spatial land capability classes map for this area are illustrated in Fig (4). The areas of these classes are shown in Table (8) which indicated that, about $4.52 \%$ of the studied soils have a Good (C2) capability class, $92.18 \%$ have a Fair (C3) capability class and the rest (3.3\%) are considered as a poor (C4) one.

Table (5): Available macronutrients as an indicator for the studied soils fertility.

\begin{tabular}{|c|c|c|c|c|c|c|}
\hline \multirow{2}{*}{ 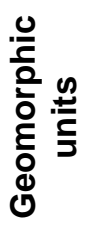 } & \multirow[b]{2}{*}{ Landform } & \multirow{2}{*}{$\begin{array}{l}0 \\
2 \\
0 \\
0 \\
\frac{0}{0} \\
\vdots \\
0\end{array}$} & \multirow{2}{*}{ 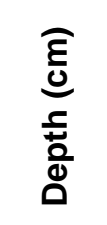 } & \multicolumn{3}{|c|}{ Macronutrients } \\
\hline & & & & $\mathbf{N}$ & $\mathbf{P}$ & K \\
\hline \multirow{6}{*}{ 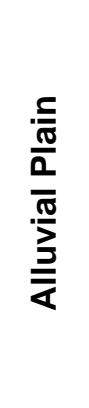 } & \multirow{2}{*}{ Low Terraces } & 1 & $0-30$ & 35.14 & 12.01 & 563.47 \\
\hline & & 2 & $0-30$ & 42.5 & 10.1 & 231.17 \\
\hline & \multirow{2}{*}{ Moderate Terraces } & 3 & $0-30$ & 13.37 & 9.02 & 93.91 \\
\hline & & 4 & $0-30$ & 13.5 & 8.01 & 151.70 \\
\hline & \multirow{2}{*}{ High Terraces } & 5 & $0-30$ & 15.65 & 9.02 & 36.12 \\
\hline & & 6 & $0-30$ & 13.25 & 8.01 & 93.91 \\
\hline \multirow{6}{*}{$\begin{array}{l}\frac{5}{\frac{\pi}{0}} \\
\frac{0}{\frac{0}{0}} \\
\frac{0}{0}\end{array}$} & \multirow{3}{*}{ Low Terraces } & 7 & $0-30$ & 19.5 & 10 & 137.25 \\
\hline & & 8 & $0-25$ & 20.22 & 10.1 & 173.37 \\
\hline & & 9 & $0-30$ & 22.5 & 11.05 & 296.18 \\
\hline & \multirow{3}{*}{ High Terraces } & 10 & $0-50$ & 15.15 & 8.21 & 166.15 \\
\hline & & 11 & $0-30$ & 13.5 & 8.01 & 72.24 \\
\hline & & 12 & $0-20$ & 13.6 & 9.02 & 216.73 \\
\hline \multirow{7}{*}{$\begin{array}{l}\frac{5}{\pi} \\
\frac{\pi}{\alpha} \\
\frac{5}{\sigma} \\
\frac{\pi}{0} \\
\frac{d}{<}\end{array}$} & \multirow{3}{*}{ Low Terraces } & 13 & $0-20$ & 11.12 & 8.01 & 130.03 \\
\hline & & 14 & $0-30$ & 9.82 & 7.21 & 144.48 \\
\hline & & 15 & $0-30$ & 13.72 & 9.02 & 202.27 \\
\hline & Moderate Terraces & 16 & $0-40$ & 17.6 & 10.01 & 158.93 \\
\hline & \multirow{3}{*}{ High Terraces } & 17 & $0-60$ & 21.2 & 11.05 & 180.60 \\
\hline & & 18 & $0-38$ & 23.23 & 11.21 & 368.42 \\
\hline & & 19 & $0-25$ & 17.64 & 9.02 & 288.96 \\
\hline
\end{tabular}


M.S. Amira, et al.,

Table (6): The main irrigation water properties.

\begin{tabular}{|c|c|c|c|c|c|c|c|c|c|c|c|c|c|}
\hline \multirow{2}{*}{ 吕 } & \multirow[b]{2}{*}{ Landform } & \multirow[b]{2}{*}{$\begin{array}{c}\text { Irrigation } \\
\text { water } \\
\text { sample } \\
\text { No. }\end{array}$} & \multirow[b]{2}{*}{$\mathrm{pH}$} & \multirow[b]{2}{*}{$\begin{array}{c}\text { EC } \\
\mathrm{ds} / \mathrm{m}\end{array}$} & \multicolumn{4}{|c|}{ Cations (meq / L) } & \multicolumn{3}{|c|}{ Anions (meq/L) } & \multirow[b]{2}{*}{$\begin{array}{c}\text { SAR } \\
\%\end{array}$} & \multirow[b]{2}{*}{$\begin{array}{c}\text { Soluble } \\
\text { boron } \\
\text { mg/L }\end{array}$} \\
\hline & & & & & $\mathrm{Ca}^{+2}$ & $\mathrm{Mg}^{+2}$ & $\mathrm{Na}^{+}$ & $\mathrm{K}^{+}$ & $\begin{array}{c}\mathrm{CO}_{3}^{-2} \& \\
\mathrm{HCO}_{3}\end{array}$ & $\mathrm{CL}^{-}$ & $\mathrm{SO}_{4}^{-2}$ & & \\
\hline \multirow{6}{*}{$\begin{array}{l}\text { Alluvial } \\
\text { Plain }\end{array}$} & \multirow{2}{*}{$\begin{array}{c}\text { Low } \\
\text { Terraces }\end{array}$} & 1 & 7.9 & 0.37 & 1.25 & 0.68 & 1.60 & 0.17 & 0.35 & 2.80 & 0.55 & 1.63 & 0.10 \\
\hline & & 2 & 7.8 & 0.50 & 1.30 & 0.85 & 2.45 & 0.40 & 0.4 & 3.11 & 1.49 & 2.36 & 0.08 \\
\hline & \multirow{2}{*}{$\begin{array}{l}\text { Moderate } \\
\text { Terraces }\end{array}$} & 3 & 8.1 & 1.89 & 5.80 & 5.50 & 6.25 & 1.35 & 0.52 & 11.75 & 6.63 & 2.63 & 0.09 \\
\hline & & 4 & 8.0 & 1.36 & 4.03 & 3.22 & 5.37 & 0.98 & 0.48 & 7.95 & 5.17 & 2.82 & 0.09 \\
\hline & \multirow{2}{*}{$\begin{array}{c}\text { High } \\
\text { Terraces }\end{array}$} & 5 & 7.8 & 2.00 & 7.65 & 4.65 & 7.13 & 0.57 & 0.61 & 12.12 & 7.27 & 2.88 & 0.09 \\
\hline & & 6 & 8.0 & 0.90 & 2.90 & 2.25 & 3.50 & 0.35 & 0.37 & 5.75 & 2.88 & 2.18 & 0.08 \\
\hline \multirow{6}{*}{ Pediplain } & \multirow{3}{*}{$\begin{array}{c}\text { Low } \\
\text { Terraces }\end{array}$} & 7 & 7.8 & 1.65 & 5.61 & 4.15 & 5.73 & 1.01 & 0.60 & 10.88 & 5.02 & 2.59 & 0.08 \\
\hline & & 8 & 7.8 & 1.65 & 5.61 & 4.15 & 5.73 & 1.01 & 0.60 & 10.88 & 5.02 & 2.59 & 0.07 \\
\hline & & 9 & 7.8 & 1.92 & 7.37 & 3.67 & 7.75 & 0.41 & 0.85 & 12.12 & 6.23 & 3.30 & 0.08 \\
\hline & \multirow{3}{*}{$\begin{array}{c}\text { High } \\
\text { Terraces }\end{array}$} & 10 & 7.8 & 2.63 & 8.95 & 4.18 & 12.44 & 0.73 & 1.28 & 16.33 & 8.69 & 4.86 & 0.07 \\
\hline & & 11 & 8.2 & 0.79 & 1.85 & 0.78 & 3.80 & 1.47 & 0.42 & 4.28 & 3.20 & 3.31 & 0.08 \\
\hline & & 12 & 8.2 & 0.79 & 1.85 & 0.78 & 3.80 & 1.47 & 0.42 & 4.28 & 3.20 & 3.31 & 0.08 \\
\hline \multirow{5}{*}{$\begin{array}{c}\text { Aeolian } \\
\text { Plain }\end{array}$} & $\begin{array}{c}\text { Low } \\
\text { Terraces }\end{array}$ & 14 & 7.9 & 0.99 & 1.48 & 0.46 & 2.28 & 5.68 & 0.27 & 3.95 & 5.68 & 2.31 & 0.09 \\
\hline & \begin{tabular}{|c|} 
Moderate \\
Terraces \\
\end{tabular} & 16 & 7.8 & 4.60 & 14.05 & 8.81 & 20.2 & 2.94 & 1.78 & 30.40 & 13.82 & 5.97 & 0.09 \\
\hline & \multirow{3}{*}{$\begin{array}{c}\text { High } \\
\text { Terraces }\end{array}$} & 17 & 7. 8 & 1.42 & 4.20 & 3.25 & 6.20 & 0.55 & 0.37 & 13.40 & 0.43 & 3.21 & 0.09 \\
\hline & & 18 & 8.0 & 3.10 & 9.33 & 5.15 & 13.78 & 2.74 & 1.23 & 19.92 & 9.85 & 5.12 & 0.08 \\
\hline & & 19 & 8.1 & 0.58 & 1.67 & 1.05 & 2.48 & 0.60 & 0.25 & 3.35 & 2.20 & 2.13 & 0.09 \\
\hline
\end{tabular}

Table (7): Land capability indices and classes for the study area.

\begin{tabular}{|c|c|c|c|c|}
\hline \multirow{2}{*}{ Geomorphic units } & \multirow{2}{*}{ Landform } & \multirow{2}{*}{$\begin{array}{l}\text { Soils of } \\
\text { profile }\end{array}$} & \multicolumn{2}{|c|}{ Land Capability } \\
\hline & & & indices & classes \\
\hline \multirow{6}{*}{ Alluvial Plain } & \multirow{2}{*}{ Low Terraces } & 1 & 69.36 & C2 (Good) \\
\hline & & 2 & 63.14 & C2 (Good) \\
\hline & \multirow{2}{*}{ Moderate Terraces } & 3 & 41.01 & C3 (fair) \\
\hline & & 4 & 45.55 & C3 (fair) \\
\hline & \multirow{2}{*}{ High Terraces } & 5 & 36.74 & C4 (poor) \\
\hline & & 6 & 44.56 & C3 (fair) \\
\hline \multirow{6}{*}{ Pediplain } & \multirow{3}{*}{ Low Terraces } & 7 & 47.3 & C3 (fair) \\
\hline & & 8 & 54.19 & C3 (fair) \\
\hline & & 9 & 51.79 & C3 (fair) \\
\hline & \multirow{3}{*}{ High Terraces } & 10 & 43.02 & C3 (fair) \\
\hline & & 11 & 50.77 & C3 (fair) \\
\hline & & 12 & 53.4 & C3 (fair) \\
\hline \multirow{7}{*}{ Aeolian Plain } & \multirow{3}{*}{ Low Terraces } & 13 & 45.04 & C3 (fair) \\
\hline & & 14 & 43.37 & C3 (fair) \\
\hline & & 15 & 53.09 & C3 (fair) \\
\hline & Moderate Terraces & 16 & 28.9 & C4 (poor) \\
\hline & \multirow{3}{*}{ High Terraces } & 17 & 47.01 & C3 (fair) \\
\hline & & 18 & 41.04 & C3 (fair) \\
\hline & & 19 & 51.97 & C3 (fair) \\
\hline
\end{tabular}




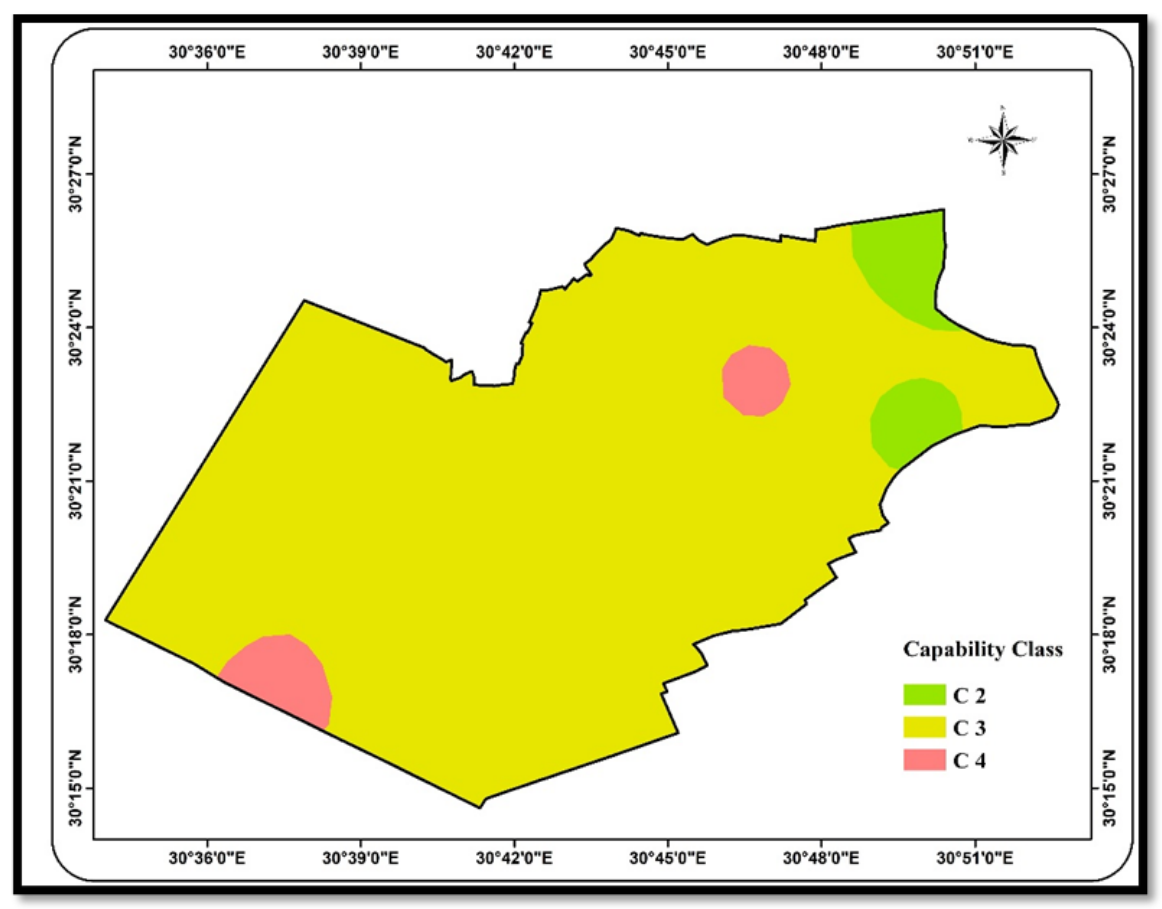

Fig (4): Land capability classes map of the studied area.

Table (8): Areas of land capability classes for the studied soils.

\begin{tabular}{|c|c|c|c|}
\hline Capability classes & Area $(\mathrm{km} 2)$ & Area (Fed) & $\%$ \\
\hline C2 (Good) & 15.94 & 3795.83 & 4.52 \\
\hline C3 (Fair) & 325.67 & 77540.51 & 92.18 \\
\hline C4 (poor) & 11.68 & 2781.58 & 3.30 \\
\hline Total & 353.29 & 84117.92 & 100 \\
\hline
\end{tabular}

\section{REFERANCES}

Burt, Rebecca and Soil Survey Staff (2014). Kellogg Soil Survey Laboratory Methods Manual, Soil Survey Investigations Report No. 42, Version 5.0, Kellogg Soil Survey Laboratory, National Soil Survey Center, Natural Resources Conservation Service, USDA, Lincoln, Nebraska, USA.

Dawoud, M.A., M.M. Darwish and M.M. ElKady (2005). GIS-based groundwater management model for Western Nile Delta. Water Resources. Manag. 19: 585-604.Dehaan, R, L. and Taylor, G, R. (2003): Image-derived spectral endmembers as indicators of salinization. International Journal of Remote Sensing, 24(4): 775-794.
Dehaan, R. L. and G. R. Taylor (2003). Image-derived spectral endmembers as indicators of salinization. International Journal of Remote Sensing, 24(4): 775-794.

Dobos, E., B. Norman, W. Bruee, M. Luca., J. Chris and M. Erika (2002). The use of DEM and satellite images for regional scale soil database. Proceedings of the 17th World Congress of Soil Science, Bangkok.

ESRI "Environmental Systems Research Institute" (2003). Using ArcGIS Geostatistical Analyst. Environmental Systems Research Institute (ESRI) Press, Redlands, California.

ESRI "Environmental Systems Research Institute" (2014). Arc Map Version 10.1 User Manual. ESRI, 380 New York 
Street, Redlands, California, 923738100, USA.

El-Maaz, Enshrah, I.M. (1997). Classification and evaluation of some soils in Western side of Nile Delta. M.Sc. Thesis, Fac. of Agric., Menoufia Univ., Egypt.

FAO (2006). Guidelines for soil profile description. Soil Res. Dev. and Co. Serv., Land and Water Dev. Div., Rome, Italy.

FAO (1977). Soil map of the world 1 : 5000 000, Volume VI Africa, FAO, UNSCO, Paris.

Ismail, H. A., E. M. El-Zahaby and M. E. ElFayoumy (1994). A modify approach for land evaluation under arid condition. II. Applications. J. Agric. Sci., Mansoura Univ., 19 (10): 34973513.

Ismail, H.A., M.H. Bahnassy and O.R. Abd El-Kawy (2005). Integrating GIS and modelling for agricultural land suitability evaluation at East Wadi ElNatrun, Egypt. Egyptian J Soil Sci., 45: 297-322.

Ismail, M., Y. A. Nasr and Y. Kotb (2010). Assessment of Soils of Wadi El-Natrun Area, Egypt. Using Remote Sensing and GIS Techniques. Journal of American Science., 6(10): 195-206.

Lillesand, T. M. and R. W. Kiefer (2007). Remote Sensing and Image Interpretation. 5th Ed. Paper back. John Wiley, New York.

RIGW and IWACO (1991). Monitoring and controlled groundwater pollution in the Nile Delta and adjacent desert areas, EI Kanater El Khairia, Egypt. TN77.0130091-12.

Soil Survey Staff (1994): Keys to Soil Taxonomy, 6th Edition Soil Conservation Service, USDA.

Soil Survey Staff (2014). Keys to Soil Taxonomy, 11 $1^{\text {th }}$ Ed., USDA, NRCS, Pocahontas Press, Inc., Blacksburg, Virginia, USA.

USDA-NRCS (1997). Soil climate map, Soil Science Division, World Soil Resources, United States Department of Agriculture \& Natural Recourses Conservation Service, Washington D.C.

Zinck, J.A. and C.R. Valenzuela (1990). Soil Geographic Database: Structure and Application Examples. ITC journal, 3: 270. 
خصائص وتقسيم وتقييم أراضي المنظقة الواقعة جنوب شرق مدينة السادات، محافظة المنوفية، مصر

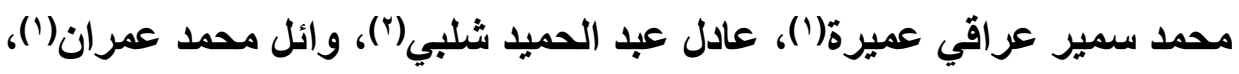

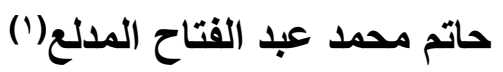

(1) (1) قسم علوم الأراضي - كلية الززاعة - جامعة المنوفية

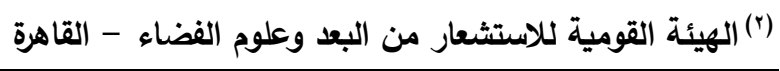

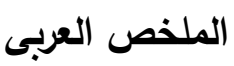
أجري هذا البحث خلال 9 أب ب بهاف دراسة الخصائص الجيومرفولوجية والبيدولوجية وكنلك تقسيم وتقييم أراضي المنطقة

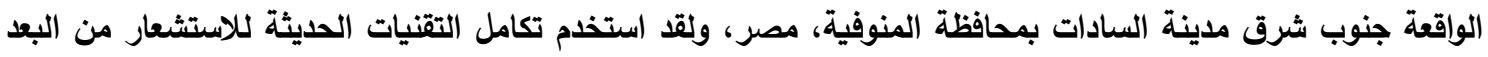

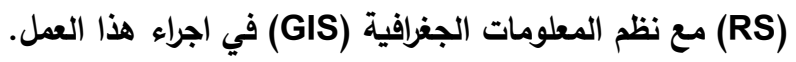
ولقد أوضحت الخريطة الجيومرفولوجية الناتجة من معالجة وتفسير الصور الجئة الجوية أن منطقة الاراسة تتميز بوجود ثلاث

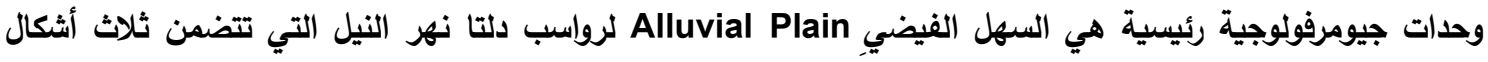

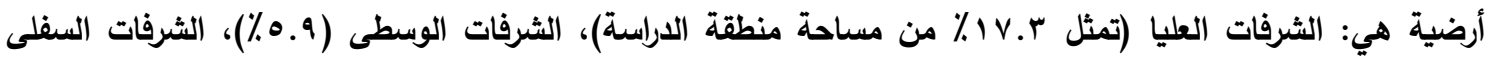

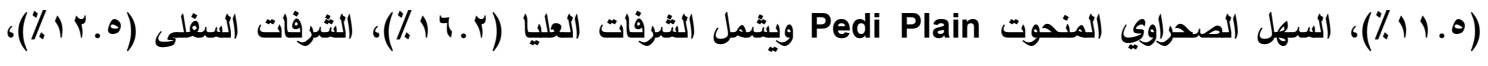

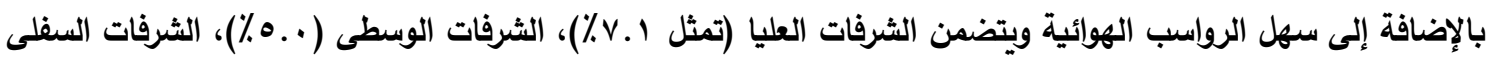

ولقد تم تحديد مواقع وحفر تسعة عشر قطاعاً أرضياً لتمثل أراضي تلك الأثكال الأرضية، ودرست وسجلت الملامح

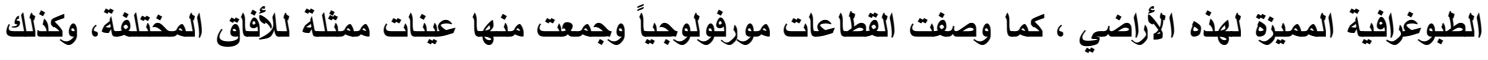

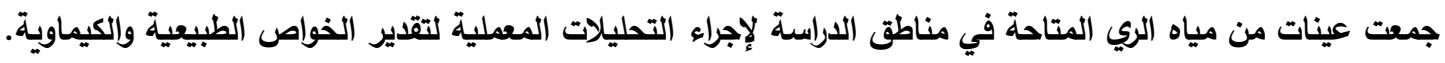

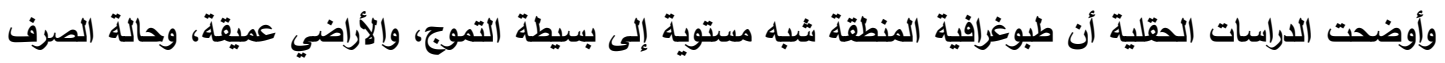

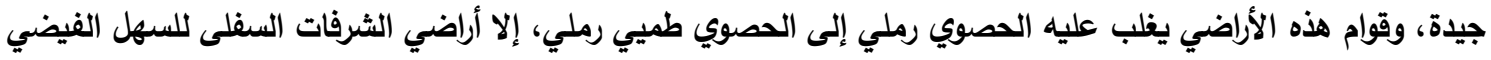

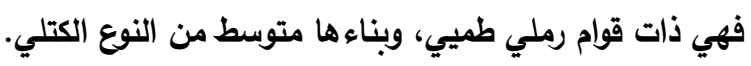

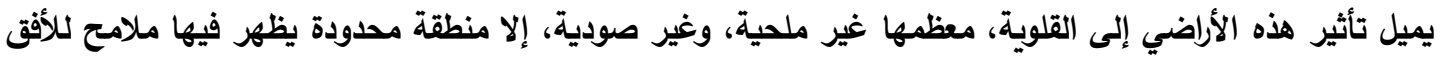

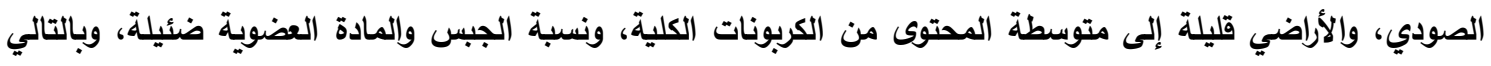
السعة التبادلية الكاتيونية منففضة.

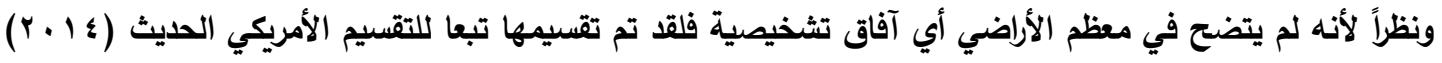

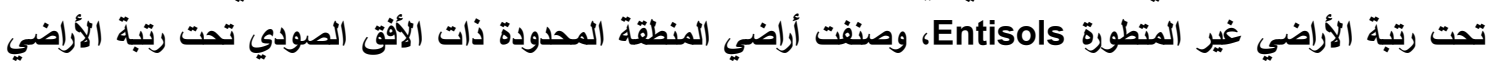

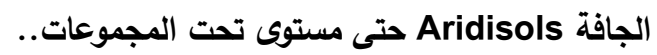

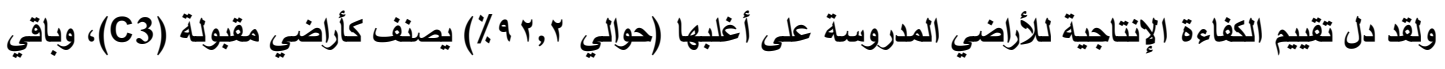

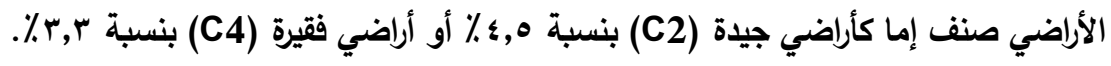
الكلمات الدالة: الاستثعار من البعد، نظم المعلومات الجغرافية، الوحدات الجيومورفولوجية، تقسيم الأراضي، تقييم الأراضي.

كلية الزراعة - جامعة المنوفية كلية الزراعة - جامعة المنوفية
السادة المحكمين

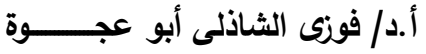
أ.د/أد/الحسينى عبدالغفار أبوحسين أبو عجئ 
M.S. Amira, et al., 